\title{
Characterization of Apicomplexan Amino Acid Transporters (ApiATs) in the Malaria Parasite Plasmodium falciparum
}

\author{
(D) Jan Stephan Wichers, ${ }^{a, b, c}$ (D) Carolina van Gelder, ${ }^{a, b, c *}$ (D) Gwendolin Fuchs, ${ }^{a, b, c s}$ (D) Julia Mareike Ruge, ${ }^{a, b, c}$ (D) Emma Pietsch, ${ }^{a, b, c}$ \\ (D) Josie L. Ferreira, ${ }^{a, d}$ (D) Soraya Safavi, ${ }^{a, b, c}$ Heidrun von Thien, ${ }^{a, b, c}$ (D) Paul-Christian Burda, ${ }^{a, b, c}$ (D) Paolo Mesén-Ramirez, \\ (D) Tobias Spielmann, ${ }^{b}$ (D) Jan Strauss, ${ }^{a, b, c, e}$ (D) Tim-Wolf Gilberger, ${ }^{a, b, c}$ (D) Anna Bachmann ${ }^{a, b, c, f}$
}

aCentre for Structural Systems Biology, Hamburg, Germany

bBernhard Nocht Institute for Tropical Medicine, Hamburg, Germany

cBiology Department, University of Hamburg, Hamburg, Germany

dHeinrich-Pette-Institute, Leibniz Institute for Experimental Virology, Hamburg, Germany

eGEOMAR Helmholtz Centre for Ocean Research Kiel, Kiel, Germany

fGerman Center for Infection Research (DZIF), partner site Hamburg-Borstel-Lübeck-Riems, Germany

Carolina van Gelder and Gwendolin Fuchs contributed equally to this work.

ABSTRACT During the symptomatic human blood phase, malaria parasites replicate within red blood cells. Parasite proliferation relies on the uptake of nutrients, such as amino acids, from the host cell and blood plasma, requiring transport across multiple membranes. Amino acids are delivered to the parasite through the parasite-surrounding vacuolar compartment by specialized nutrient-permeable channels of the erythrocyte membrane and the parasitophorous vacuole membrane (PVM). However, further transport of amino acids across the parasite plasma membrane (PPM) is currently not well characterized. In this study, we focused on a family of Apicomplexan amino acid transporters (ApiATs) that comprises five members in Plasmodium falciparum. First, we localized four of the P. falciparum ApiATs (PfApiATs) at the PPM using endogenous green fluorescent protein (GFP) tagging. Next, we applied reverse genetic approaches to probe into their essentiality during asexual replication and gametocytogenesis. Upon inducible knockdown and targeted gene disruption, a reduced asexual parasite proliferation was detected for PfApiAT2 and PFApiAT4. Functional inactivation of individual PfApiATs targeted in this study had no effect on gametocyte development. Our data suggest that individual PfApiATs are partially redundant during asexual in vitro proliferation and fully redundant during gametocytogenesis of $P$. falciparum parasites.

IMPORTANCE Malaria parasites live and multiply inside cells. To facilitate their extremely fast intracellular proliferation, they hijack and transform their host cells. This also requires the active uptake of nutrients, such as amino acids, from the host cell and the surrounding environment through various membranes that are the consequence of the parasite's intracellular lifestyle. In this paper, we focus on a family of putative amino acid transporters termed ApiAT. We show expression and localization of four transporters in the parasite plasma membrane of Plasmodium falciparum-infected erythrocytes that represent one interface of the pathogen to its host cell. We probed into the impact of functional inactivation of individual transporters on parasite growth in asexual and sexual blood stages of $P$. falciparum and reveal that only two of them show a modest but significant reduction in parasite proliferation but no impact on gametocytogenesis, pointing toward dispensability within this transporter family.
Citation Wichers JS, van Gelder C, Fuchs G Ruge JM, Pietsch E, Ferreira JL, Safavi S, von Thien H, Burda P-C, Mesén-Ramirez P, Spielmann T, Strauss J, Gilberger T-W, Bachmann A. 2021. Characterization of apicomplexan amino acid transporters (ApiATs) in the malaria parasite Plasmodium falciparum. mSphere 6:e00743-21. https://doi .org/10.1128/mSphere.00743-21.

Editor Margaret Phillips, University of Texas Southwestern

Copyright $\odot 2021$ Wichers et al. This is an open-access article distributed under the terms of the Creative Commons Attribution 4.0 International license.

Address correspondence to Tim-Wolf Gilberger, gilberger@bnitm.de, or Anna Bachmann, bachmann@bnitm.de.

*Present address: Carolina van Gelder, University of Duisburg-Essen, Essen, Germany. §Present address: Gwendolin Fuchs, Karolinska Institutet, Stockholm, Sweden

Received 7 September 202

Accepted 24 October 2021

Published 10 November 2021 
alaria parasites replicate within human erythrocytes during the asexual blood phase, which is responsible for the symptoms of the disease. Although Plasmodium falciparum is able to synthesize some amino acids de novo (1-6) during the intraerythrocytic development, the parasite relies heavily on amino acid acquisition from its host. Amino acids are derived either from the digestion of hemoglobin endocytosed from the infected erythrocyte $(1,7)$ or from the uptake of free amino acids from the blood plasma (1). Both processes are important for efficient parasite growth (8). The parasite is able to import all 20 naturally occurring $\alpha$-amino acids from the external medium and uses them for its own protein synthesis (9-12). The import of isoleucine (13-15) - and for some $P$. falciparum strains methionine also (13) - is crucial for the survival of the parasite, as adult human hemoglobin lacks isoleucine. Accordingly, an increase in the permeability to a range of amino acids has been reported for erythrocytes upon Plasmodium infection $(10,11,16,17)$. This is mainly mediated via the new permeability pathways (NPPs) established by the parasite within the membrane of the infected erythrocyte $(16,17)$. While the subsequent transport across the parasitophorous vacuole membrane (PVM) is linked to nutrient-permeable channel activity (1821), the molecular machinery responsible for the further transport across the parasite plasma membrane (PPM) is not well defined. Neutral amino acids like isoleucine and methionine traverse the PPM more rapidly than anionic and cationic amino acids, which may be coupled to the transport of other substrates like $\mathrm{H}^{+}$or $\mathrm{Na}^{+}(16,22)$. However, neither the membrane transporters nor the exact mechanism(s) by which amino acids cross the PPM have been characterized so far $(8,22)$.

The $P$. falciparum transportome is predicted to be encoded by 144 genes (23), of which at least 11 are classified as putative amino acid transporters $(23,24)$. Five of these putative amino acid transporters belong to the Apicomplexan amino acid transporter (ApiAT) $(25,26)$ family. This Apicomplexan-specific family of transmembrane transporters can be subdivided into the 11 subfamilies ApiAT1 to ApiAT11. Some of the subfamilies are lineage specific. For instance, ApiAT4, ApiAT8, ApiAT9, and ApiAT10 are present only in the genomes of Plasmodium spp. $(1,25)$. Others, such as the most ancient variant ApiAT2, can be found in many different Apicomplexan parasites (25). The main feature of the ApiAT family is the possession of multiple, typically 12 , transmembrane domains characteristic of solute transporters with a signature sequence between transmembrane domains 2 and 3 (25). This classifies them as members of the major facilitator superfamily (MFS) $(24,27,28)$. However, overall they have limited sequence similarity to other known eukaryotic or prokaryotic transporters (25).

$P$. falciparum possesses 5 of the 11 ApiAT subfamilies: P. falciparum ApiAT2 (PfApiAT2) (PfApiAT2/MFR4: PF3D7_0914700), PfApiAT4 (MFR5: PF3D7_1129900), PfApiAT8 (NPT1: PF3D7_0104800), PfApiAT9 (MFR2: PF3D7_0104700), and PfApiAT10 (MFR3: PF3D7_0312500), previously termed major facilitator superfamily-related transporter (MFR) or novel putative transporter (NPT). Previous work in the rodent malaria species Plasmodium berghei and the related apicomplexan parasite Toxoplasma gondii showed that $P$. berghei ApiAT8 (PbApiAT8) (or PbNPT1) and several other T. gondii ApiATs (TgApiATs) are localized at the PPM (29-32) and possess amino acid transport activity $(25,26,29-34)$. To date, only PbApiAT4 has been shown to play an important role in parasite proliferation within erythrocytes (35). Apart from this, PbApiAT8 is essential for gametocyte development $(29,30,35)$, and all variants except PbApiAT2 appear to be necessary for exflagellation of male gametocytes (35). Additionally, sporozoite development likely requires PbApiAT2, PbApiAT4, PbApiAT9, and PbApiAT10 (35), and a lack of PbApiAT2 further impairs oocyst development. Furthermore, an association of episomally overexpressed PfApiAT10-GFP (PfApiAT10 tagged with green fluorescent protein [GFP]) (termed MFR3-GFP) with the mitochondrion has been reported (36).

In this study, we characterize the localization and essentiality of the ApiAT family members of $P$. falciparum during intraerythrocytic development in order to further dissect the function of these unique transporters. 


\section{RESULTS}

$\boldsymbol{P}$. falciparum ApiATs localize at the parasite plasma membrane. The five members of the ApiAT family in $P$. falciparum show different gene expression patterns and mRNA levels during the intraerythrocytic developmental cycle (IDC) (37). While PfApiAT2 has its maximum transcript level in early ring stage parasites ( $8 \mathrm{~h}$ postinfection [hpi]) and PfApiAT4 and PfApiAT10 mRNA levels peak in late ring stage parasites (16 hpi), PfApiAT8 shows a maximum of transcripts in late stage schizonts (48 hpi) and mRNA of PfApiAT9 is almost absent during the IDC (Fig. 1A). Overall, PfApiAT2 and PfApiAT4 are most abundantly expressed on the mRNA level.

To determine their protein expression and localization, we tagged each of the five members of the PfApiAT family endogenously with GFP using the selection-linked integration (SLI) system (38). Correct integration of the corresponding targeting plasmids into the respective genomic loci was verified by PCR (see Fig. S1A in the supplemental material). Except for 3D7-ApiAT9-GFP, all generated transgenic cell lines expressed the full-length fusion protein (Fig. S1B) to a sufficient level that allowed its subcellular localization. All of them are localized at the parasite periphery (Fig. 1B). Subsequent colocalization with the episomally expressed PPM marker Lyn (39)-mCherry (38) reveals PPM localization that becomes particularly evident in free merozoites, where PPM- and PVM-localized proteins can clearly be separated (Fig. 1C).

In contrast to published data (36), we also found endogenously GFP-tagged PfApiAT10 localizing at the PPM. To reprobe into the apparent PPM localization of endogenously GFP-tagged PfApiAT10, we also overexpressed this gene as a GFP and mCherry fusion protein using two different promoters (crt [45] or ama1 [46]). This allowed us to assess the influence of the tags as well as differential expression profiles on PfApiAT10 protein localization. All cell lines showed PPM localization of the PfApiAT10 fusion proteins (Fig. 1B to E and Fig. S1). Of note, functional inactivation of PfApiAT10 did not result in conferral of drug resistance to atovaquone (Fig. S2B).

Individual PfApiATs are not essential for asexual blood stage development. To probe into the essentiality of the ApiAT family for asexual parasite proliferation, we first targeted the most abundantly expressed PfApiATs, PfApiAT2 and PfApiAT4 by conditional knockdown. Downregulation was achieved by introducing a glmS ribozyme sequence (40) before the $3^{\prime}$ untranslated region ( $3^{\prime}$ UTR) of either the apiat2 or apiat4 genomic locus (Fig. S1). The ribozyme was activated by the addition of $2.5 \mathrm{mM}$ D$(+)$-glucosamine hydrochloride (GLCN), which resulted in degradation of mRNA and therefore decreased protein levels. This was assessed and quantified by live-cell fluorescence microscopy after two cycles. GLCN treatment resulted in a decreased GFP fluorescence of $85.9 \% \pm 0.9 \%$ (mean \pm standard deviation [SD]) for 3D7-PfApiAT2-GFP or $74.8 \% \pm 14.0 \%$ for 3D7-PfApiAT4-GFP (Fig. 2A to D) and led to a moderate, but significant, reduction of parasite growth of 14.0 to $20.7 \%$ compared to 3D7 parasites cultured with GLCN (Fig. 2E). These data indicate that PfApiAT2 and PfApiAT4 play a role in efficient blood cell proliferation but imply that they might be nonessential. Therefore, we targeted these genes with deletion constructs using the SLI system (38) that led to the expression of severely truncated versions of the ApiATs. In this targeted gene disruption (TGD) approach, we also included PfApiAT8 and PfApiAT10 (Fig. S1C).

Imaging of the TGD cell lines revealed a more diffuse but still membrane-associated GFP signal (Fig. 3A to D). This might be due to the remaining transmembrane domains of the truncated PfApiAT mutants; however, our approach deleted at least three quarters of their predicted transmembrane (TM) domains and thus most likely abolished transporter activity. In concordance with the inducible knockdown data, functional inactivation by truncation of PfApiAT2 and PfApiAT4 in the corresponding transgenic cell lines (3D7-ApiAT2-TGD and 3D7-ApiAT4-TGD) led to a moderate decrease of parasite proliferation of $20.2 \% \pm 3.2 \%$ and $19.8 \% \pm 8.6 \%$ after two parasite replication cycles (Fig. 3E). No significant reduction of growth was observed upon disruption of PfApiAT8 and PfApiAT10 (Fig. 3E). Interestingly, cultivation in amino acid-depleted medium (approximately $90 \%$ reduced concentration) did not indicate a higher 
A

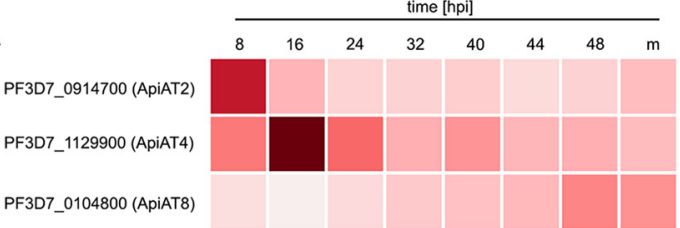

PF3D7_0104700 (ApiAT9)

PF3D7_0312500 (ApiAT10)

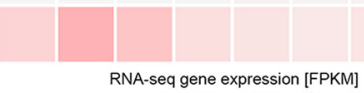

B

$\begin{array}{llll}0 & 50 \quad 100 & 150\end{array}$

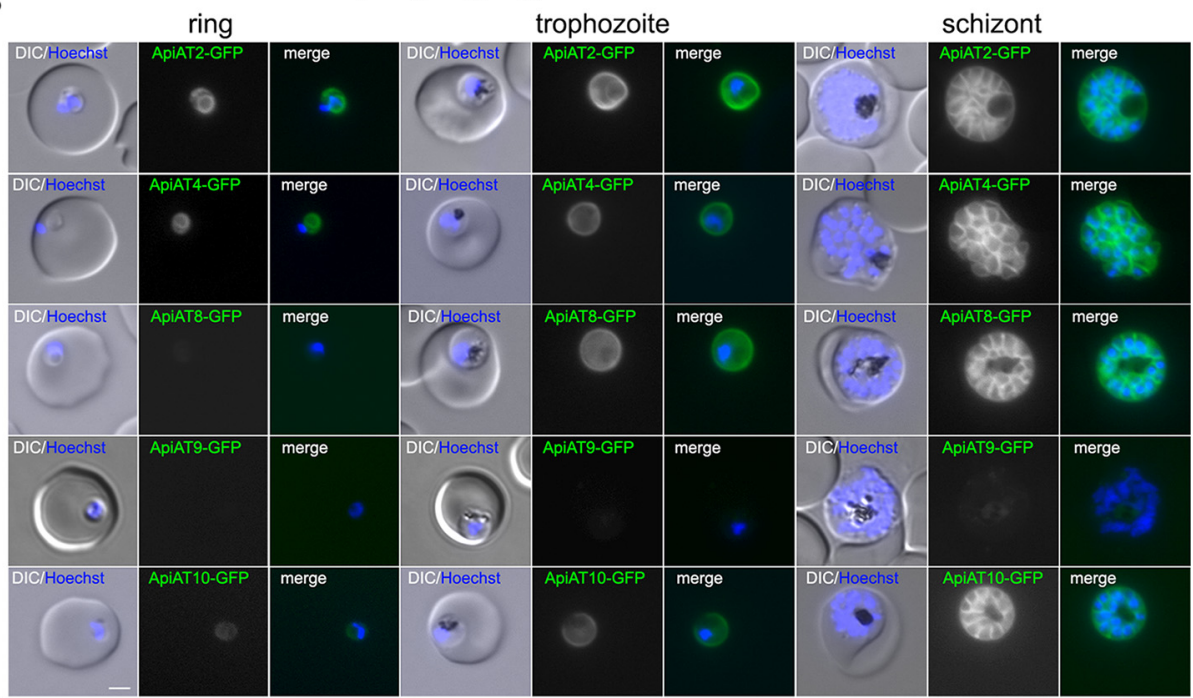

C

schizont

merozoites
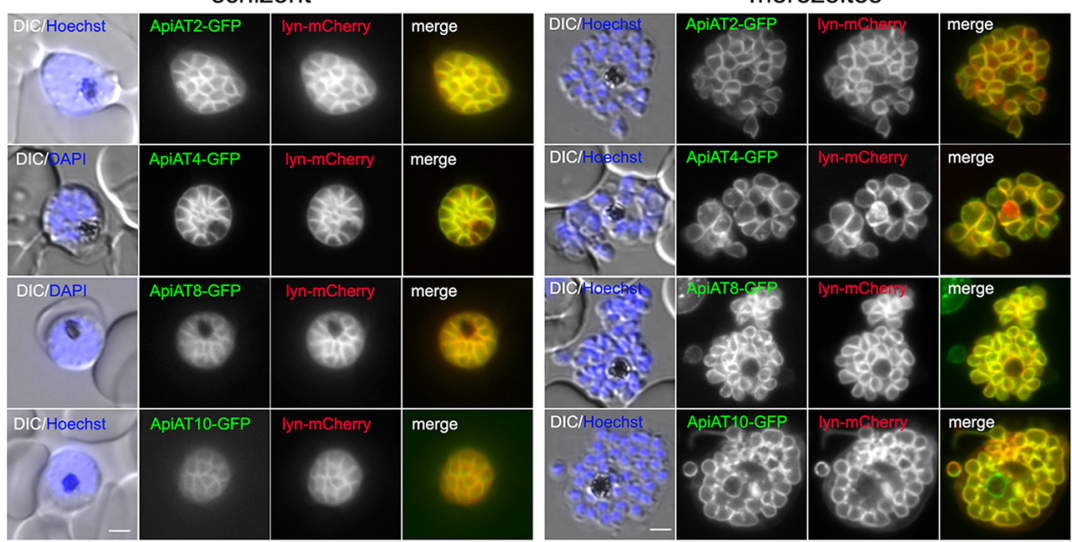

$\mathrm{D}$

$\mathrm{E}$
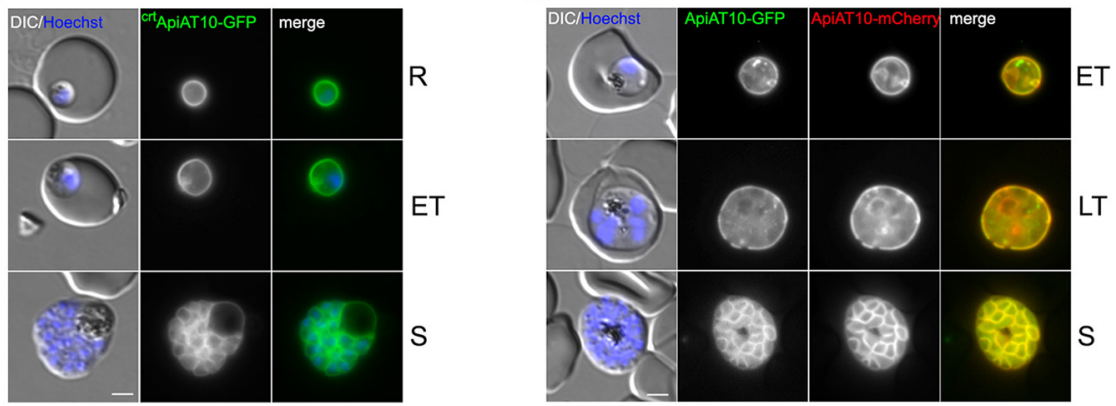

FIG 1 P. falciparum ApiATs localize to the parasite plasma membrane (PPM) during asexual blood stage development. (A) Heatmap of transcriptome sequencing (RNA-seq) gene expression profiles (37) of PfApiAT2,

(Continued on next page) 
susceptibility of any of the TGD cell lines to low amino acid concentrations compared to wild-type parasites (Fig. 3F).

To probe into potential transcriptional perturbations within this gene family due to functional inactivation of a single member, quantitative real-time PCR (qPCR) analysis was performed using RNA from four different time points during asexual blood stage replication. However, no consistent upregulation of RNA levels of other PfApiAT family members was observed in individual PfApiAT TGDs (Fig. S3).

PfApiATs are dispensable during gametocyte development. Previous data (30, 35) indicated a role of ApiAT8 during gametocyte development of the rodent malaria parasite $P$. berghei. Therefore, we reengineered the GFP-tagged gene knockdown (Fig. S4) and deletion cell lines (Fig. S5) for PfApiAT2, PfApiAT4, PfApiAT8, and PfApiAT10 in an inducible gametocyte-producing parasite line (3D7-iGP-GDV1GFP-DD [41]) using the same SLI approach. The resulting parasite lines allowed a robust, efficient, and synchronized induction of gametocytogenesis by expression of GDV1 upon addition of Shield-1 and therefore a solid basis for phenotypic analysis. First, using the C-terminal GFP tag, we confirmed expression of all four PfApiATs in gametocytes. As expected, most PfApiATs remain PPM localized during gametocytogenesis, which was additionally confirmed by the colocalization with the episomally expressed PPM marker Lyn (39)mCherry (38) (Fig. 4A to F). The exception was PfApiAT9, which showed only a faint background staining in all gametocyte stages in the 3D7-ApiAT9-GFP line (Fig. 4D). The observed prominent GFP signal at the food vacuole in gametocytes is most likely an unspecific hemozoin signal and not derived from GFP fusion proteins, as also observed in 3D7-iGP and 3D7 wild-type control parasites (Fig. S4C). Of note, PfApiAT2 was observed to be strongest expressed in early stage gametocytes and weaker in late stages (Fig. 5B and D; Fig. S4D), while PfApiAT4 showed strongest expression in late stage gametocytes (Fig. 5C and E). Next, we investigated the consequence of glmS-based conditional knockdown for PfApiAT2 and PfApiAT4 (Fig. 5A). Although 75 to $80 \%$ knockdown of PfApiAT2 or PfApiAT4 expression was achieved (Fig. 5B to E), no significant reduction in gametocytemia or aberrant gametocyte development could be detected (Fig. 5F). This was reinvestigated by targeted gene disruption. Likewise, deletion of these two genes as well as of PfApiAT8 and PfApiAT10 did not result in any measurable impairment of gametocyte development or morphology, indicating the dispensability of these individual PfApiATs for the sexual stage development of the parasite (Fig. 6 and Fig. S5).

\section{DISCUSSION}

We localized four putative amino acid transporters (PfApiAT2, PfApiAT4, PfApiAT8, and PfApiAT10) of the ApiAT family to the PPM in asexual blood stage parasites and gametocytes. Due to the low expression of PfApiAT9-GFP-in agreement with the transcript levels of apiat9 in these stages $(37,42,43)$-no conclusive localization could be delivered. The observed PPM localization of the investigated ApiATs is in concordance with the following published data. (i) The P. berghei ApiAT8 homologue was shown to be a general cationic amino acid transporter of the PPM $(29,30)$. (ii) PfApiAT8 has recently been localized to the PPM using an overexpression approach (31). (iii) Several T. gondii ApiATs (TgApiAT1, TgApiAT2, TgApiAT3-1, TgApiAT3-2, TgApiAT3-3, TgApiAT5-3, TgApiAT6-1, and TgApiAT6-3) have been located at the PPM as well $(25,29,32,33,44)$.

FIG 1 Legend (Continued)

PfApiAT4, PfApiAT8, PfApiAT9, and PfApiAT10 during the asexual blood stage development. Time points are indicated as hours postinfection (hpi) plus merozoites (m). FPKM, fragments per kilobase per million. (B) Localization of PfApiAT2-GFP, PfApiAT4-GFP, PfApiAT8-GFP, PfApiAT9-GFP, and PfApiAT10-GFP by live-cell microscopy across the IDC of 3D7 parasites. DIC, differential interference contrast. (C) Colocalization of the GFP-tagged ApiAT fusion proteins with the PPM marker protein Lyn-mCherry in schizonts and merozoites. (D) Live-cell microscopy of 3D7-crt-ApiAT10-GFP parasites across the IDC. (E) 3D7-iGP-ApiAT10-GFP/ama1-ApiAT10$m$ Cherry parasites at the trophozoite and schizont stage. Nuclei were stained with Hoechst-33342. Parasite stages are indicated as follows; ring stage (R), early trophozoite (ET), late trophozoite (LT) and schizont (S). Bars, $2 \mu \mathrm{m}$. 


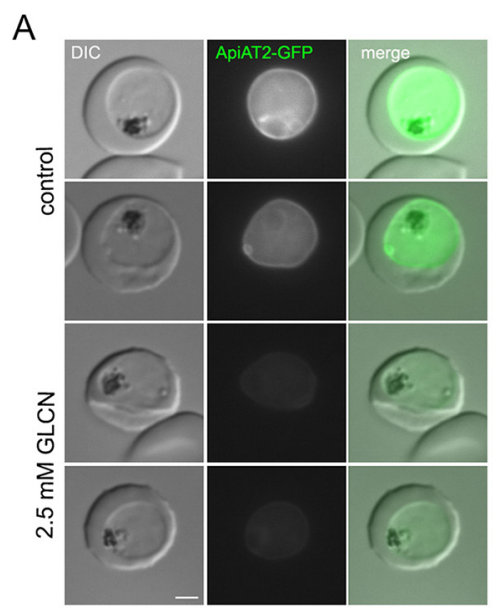

C

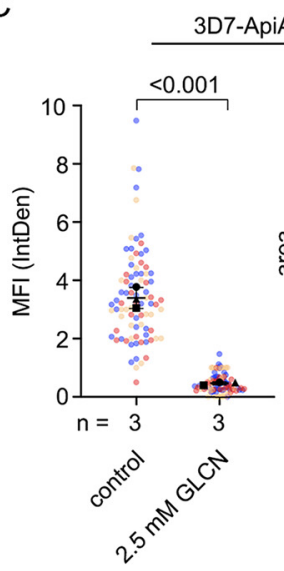

$E$

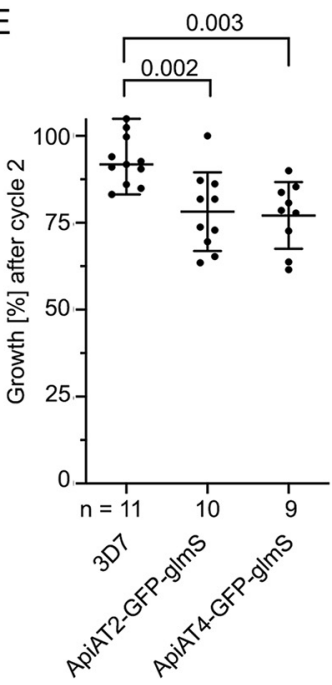

B

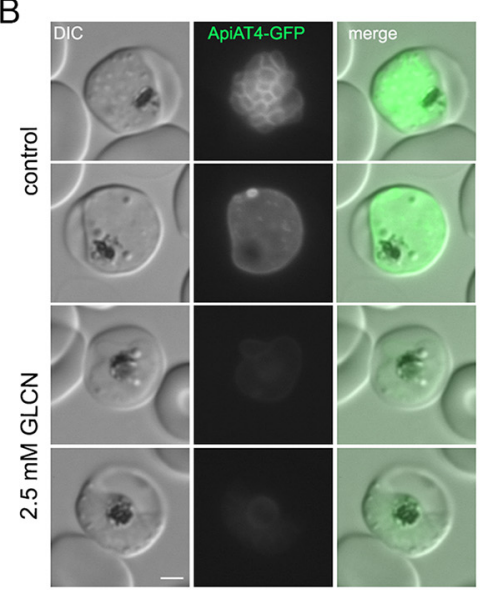

D

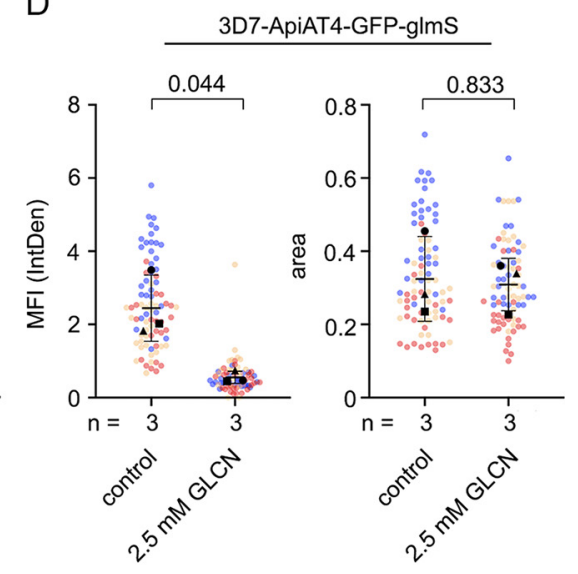

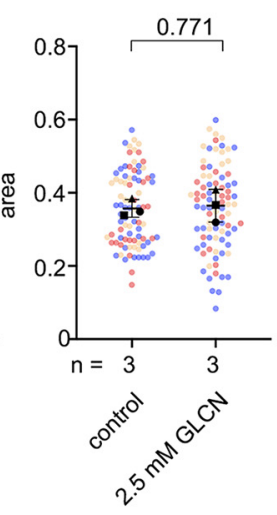

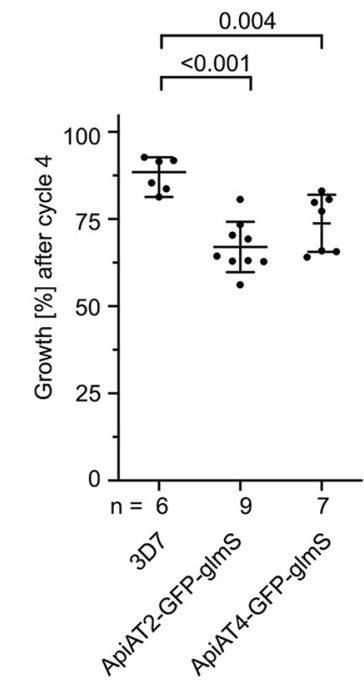

FIG 2 Conditional knockdown of PfApiAT2 and PfApiAT4 reveals a minor growth defect during asexual blood stage development. (A and B) Live-cell microscopy of 3D7-ApiAT2-GFP (A) and 3D7-ApiAT4-GFP (B) parasites, which were treated for $40 \mathrm{~h}$ with $2.5 \mathrm{mM}$ glucosamine (GLCN) or that were left untreated (control). Bars, $2 \mu \mathrm{m}$. (C and D) Quantification of knockdown efficiency by measuring mean fluorescence intensity (MFI) density (IntDen, intensity density) and parasite size (area) of 3D7-ApiAT2-GFP (C) and 3D7ApiAT4-GFP (D) parasites $40 \mathrm{~h}$ after treatment with or without $2.5 \mathrm{mM}$ glucosamine. Data are displayed as means \pm standard errors (SD) (error bars) of three independent experiments, and individual data points are displayed on a scatterplot color coded by experiments according to SuperPlots guidelines (82). $P$ values displayed were determined using a two-tailed unpaired $t$ test with Welch's correction. (E) Growth of parasites treated with or without $2.5 \mathrm{mM} \mathrm{GLCN}$ after two and four parasite replication cycles 
Recent work using episomally overexpressed PfApiAT10-GFP implied an association of this transporter with the mitochondrial membrane (36) in P. falciparum Dd2 (PfDd2) parasites. This localization differs from the observed PPM localization of endogenously GFP-tagged PfApiAT10 in both 3D7 as well as 3D7-iGP parasites (Fig. 1B and 4C; see also Fig. S2A in the supplemental material) reported in this study. We confirmed the PPM localization of PfApiAT10 by its overexpression either as a GFP or mCherry fusion using two different promoters (crt [45] and ama1 [46]). In line with that, the reported reduced sensitivity to the mitochondrial electron transport chain inhibitor atovaquone (47) upon knockout of PfApiAT10 (36) was not observed upon targeted gene disruption in our study (Fig. S2B). It is possible but appears unlikely that the reported mitochondrial association as well as reduced sensitivity to atovaquone upon overexpression is due to the different parasite strains (PfDd2 [48] versus 3D7 [this study]), given that PfDd2_apiat10 (PFDd2_030017500) has only one silent mutation at position G1080A compared to Pf3D7_apiat10 (49). Of note, PfApiAT10 is also not part of the recently published Plasmodium mitochondrial proteome (50).

During the intraerythrocytic development of the parasite, the amino acid requirements are largely covered by degradation of the globin polypeptide $(14,51)$, although - for instance-the import of isoleucine is crucial for the survival of the parasite, as $P$. falciparum lacks the canonical pathways for its biosynthesis (52) and adult human hemoglobin lacks this amino acid. Dedicated amino acid transporters could fill this gap. Therefore, we tested the impact of functional inactivation of individual PfApiATs on parasite growth in asexual and sexual blood stages of $P$. falciparum. We observed only a minor but significant reduction in parasite growth upon knockdown or gene disruption of PfApiAT2 and PfApiAT4 in asexual blood stages without compensatory upregulation of other PfApiATs on the transcriptional level, as indicated by QPCR analysis. The phenotypes are in agreement with a previously reported reduced parasite multiplication rate of $36 \%$ in PbApiAT4 knockout parasites (35). Moreover, our data are also in line with the finding that PbApiAT8 is not essential for asexual replication $(29,30,35$, 53). Additionally, functional inactivation of individual PfApiAT did not result in parasites more sensitive to reduced amino acid concentrations in the medium. The absence of profound growth effects might be explained by functional redundancy either within the ApiAT family, as recently observed for T. gondii (34), or by the presence of yet unassigned transporters capable of transporting essential amino acids such as isoleucine across the PPM. Like in T. gondii, overlapping substrate specificities and lower transport levels might be sufficient for parasite growth in vitro (34). Of note, in T. gondii, the arginine transporter TgApiAT1 has been shown to be differently regulated on the translational level in dependence of arginine mediated by an upstream open reading frame (uORF) present on the $5^{\prime}$ leader sequence of the transcript (26). A similar layer of regulation might also be present in PfApiATs. However, since Toxoplasma and Plasmodium share only one ApiAT, the likely most ancestral ApiAT2 (25), the regulatory elements as well as the general characteristics and substrates of the different PfApiATs remain largely unknown.

Interestingly, a knockout of the ApiAT8 homologue of P. berghei (PBANKA_0208300) resulted in strongly reduced number of mature gametocytes with an aberrant morphology of the remaining parasites (30) and strongly reduced exflagellation (35). In our study, functional inactivation of PfApiAT8 via targeted gene disruption had no impact on gametocyte development and morphology, which might reflect the pronounced differences in

FIG 2 Legend (Continued)

as determined by flow cytometry. Shown are relative parasitemia values, which were obtained by dividing the parasitemia of glucosamine-treated cultures by the parasitemia of the corresponding untreated ones. Displayed are means \pm SD of independent growth experiments with the number of experiments $(n)$ indicated. Adjusted $P$ values displayed were determined with a two-tailed unpaired $t$ test with Welch's correction and using the Benjamini-Hochberg correction afterwards accounting for multiple testing by comparing ApiAT2-GFP-glmS or ApiAT4-GFP-glmS cultured with $2.5 \mathrm{mM}$ GLCN to 3D7 parasites cultured with $2.5 \mathrm{mM}$ GLCN. 
A
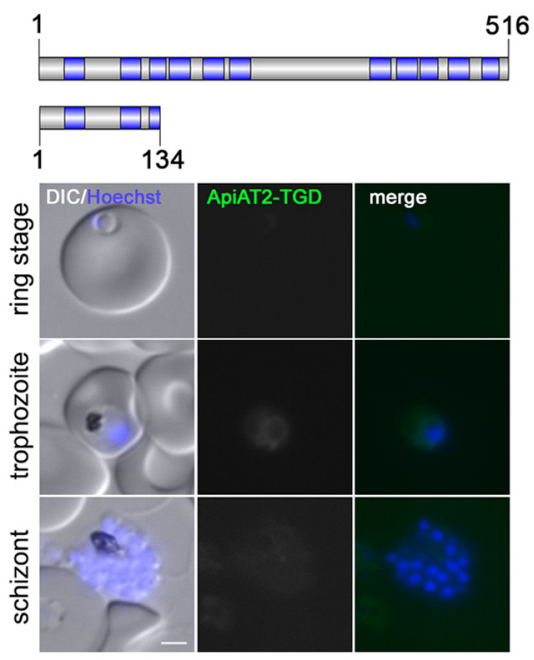

D
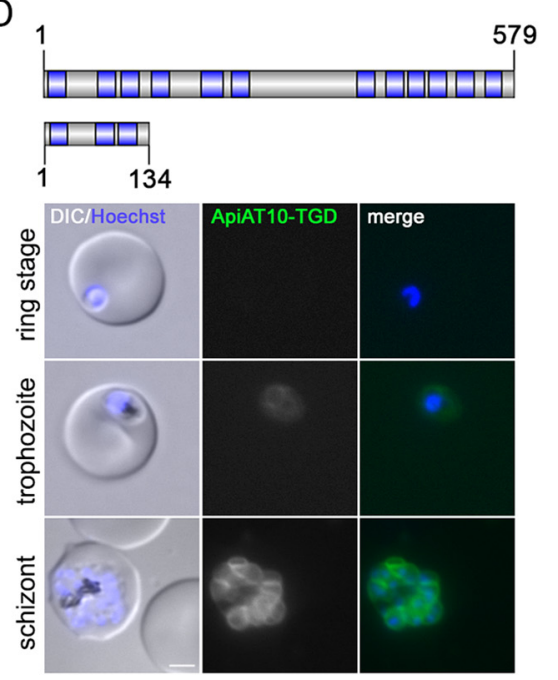

B

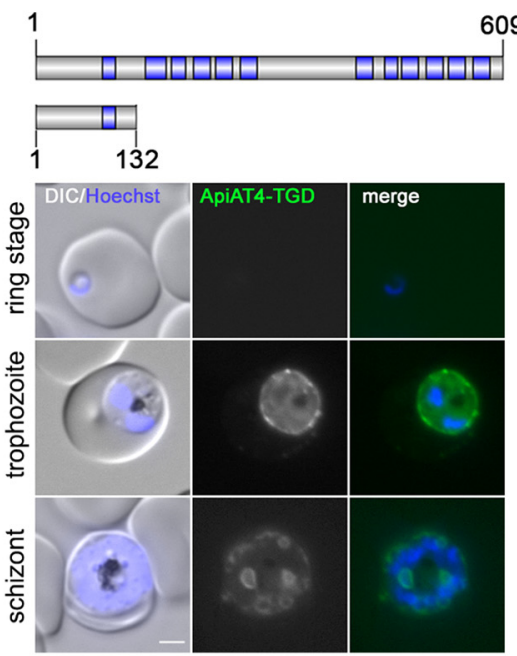

E growth in normal medium

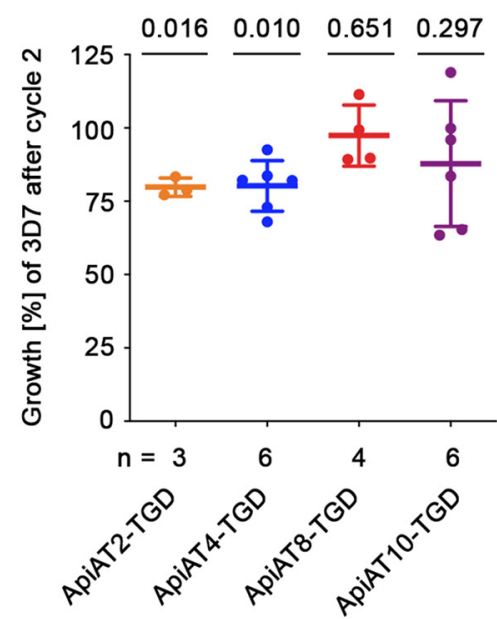

C
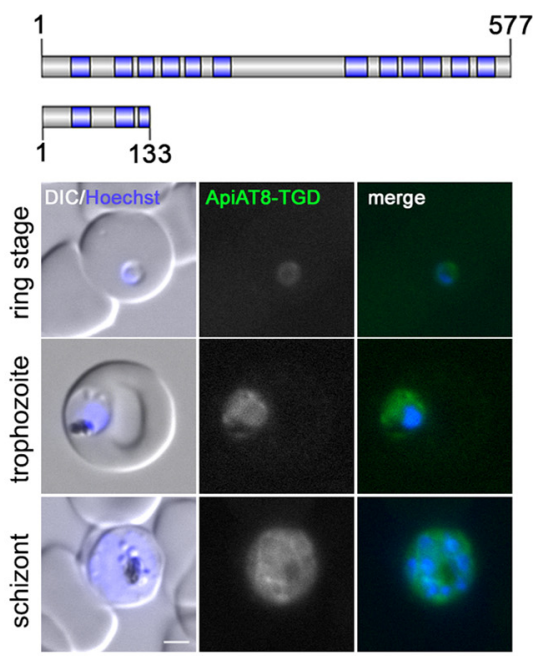

F

growth in AA depleted medium

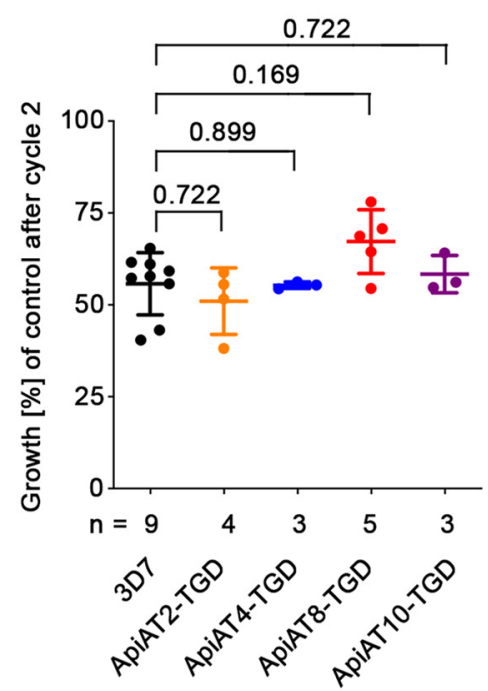

FIG 3 Targeted gene disruption (TGD) of PfApiAT2, PfApiAT4, PfApiAT8, and PfApiAT10 reveals the dispensability of PfApiATs for in vitro parasite proliferation. (A to D) Schematic representation of the full-length and truncated protein versions (top panel). Protein length (number of amino acids) and putative transmembrane protein domains (blue) are indicated. Localization of PfApiAT8-TGD-GFP (A), PfApiAT4-TGD-GFP (B), PfApiAT2-TGD-GFP (C), and PfApiAT10-TGD-GFP (D) in ring, trophozoite, and schizont stage parasites is shown in lower panels. Nuclei were stained with Hoechst-33342. Bars, $2 \mu \mathrm{m}$. (E) Growth of 3D7-ApiAT-TGD cell lines as a percentage of 3D7 parasite growth, monitored over two intracellular development cycles by flow cytometry. The number of independent growth experiments $(n)$ per 3D7-ApiAT-TGD cell line is indicated. 3D7 wild-type parasites were measured in parallel. Statistical differences were analyzed using a one-sample $t$ test with Benjamini-Hochberg correction accounting for multiple comparisons. (F) Growth of TGD and 3D7 cell lines cultivated in low-amino acid medium relative to their growth in standard medium is shown as percentage of growth after two parasite replication cycles. The number of individual growth experiments $(n)$ performed is indicated for each 3D7-ApiAT-TGD line. Additionally, 3D7 wild-type parasites were analyzed with $n=9$. No statistical differences were observed by comparing relative growth of TGD cell lines to 3D7 using a two-tailed unpaired $t$ test with Bonferroni correction. AA, amino acid.

gametocyte development between the rodent-infecting P. berghei and the human-infecting $P$. falciparum parasites (54). For future work, it will be interesting to target multiple ApiATs by gene disruption in parallel to assess their putative synergy and to probe into PfApiAT function in other fast-replicating stages of $P$. falciparum such as liver stages, for which the essentiality of several metabolic processes has recently been shown and that primarily rely on the amino acid uptake from their host (55).

\section{MATERIALS AND METHODS}

P. falciparum culture. Blood stages of $P$. falciparum 3D7 (56) were cultured in human red blood cells $(\mathrm{O}+$ or $\mathrm{B}+)$. Cultures were maintained at $37^{\circ} \mathrm{C}$ in an atmosphere of $1 \% \mathrm{O}_{2}, 5 \% \mathrm{CO}_{2}$, and $94 \% \mathrm{~N}_{2}$ using RPMI 
A

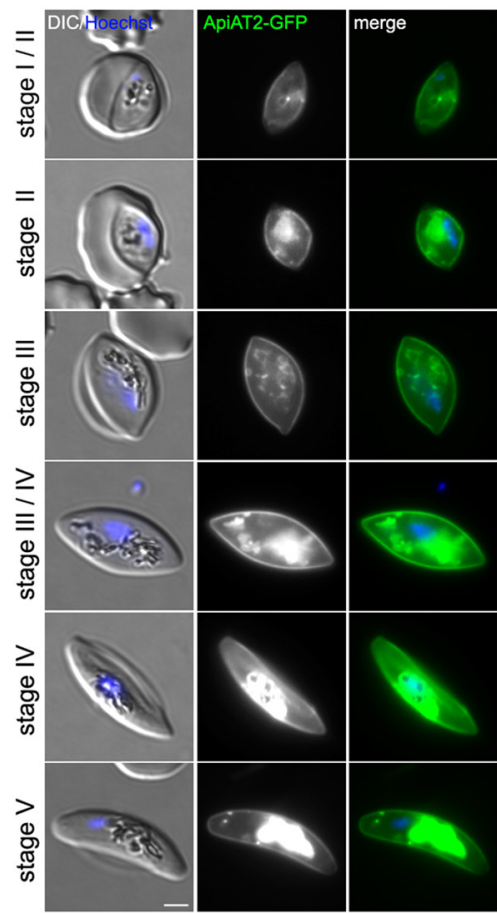

D

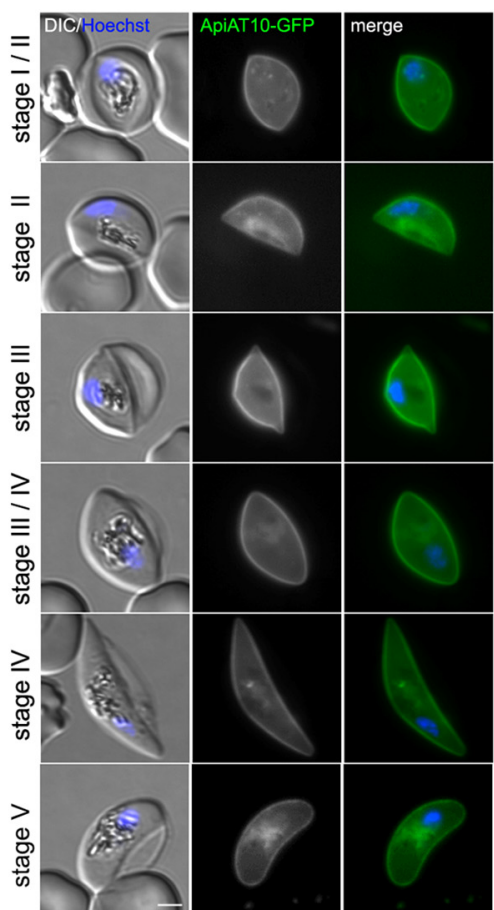

B

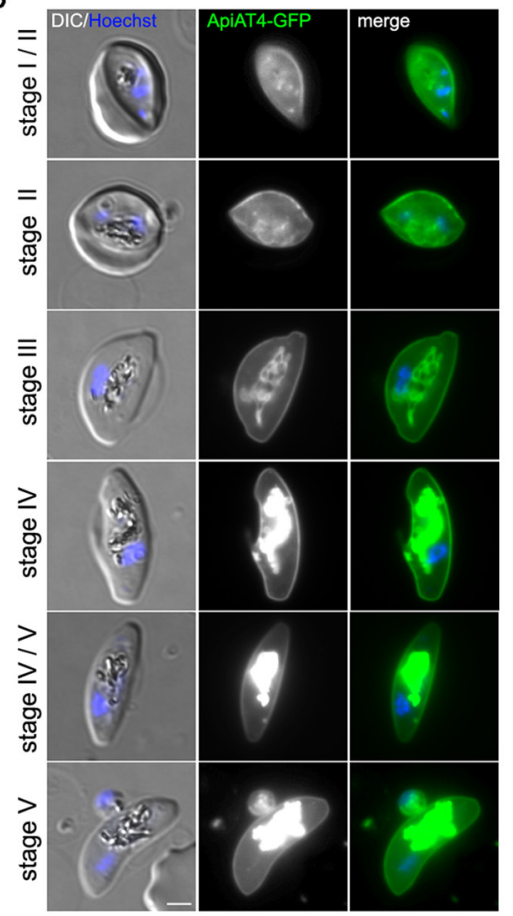

E

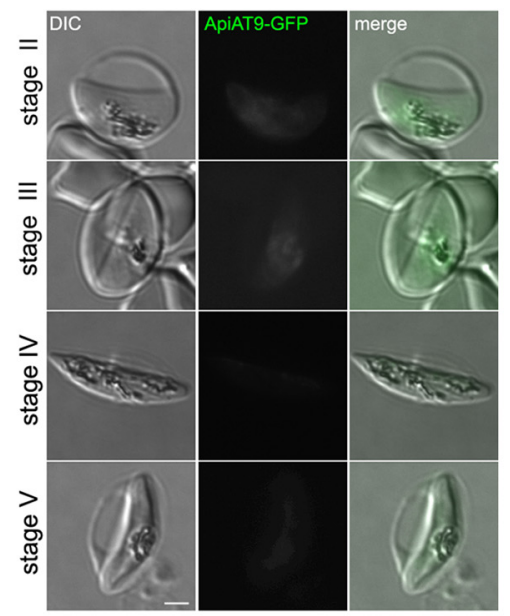

C

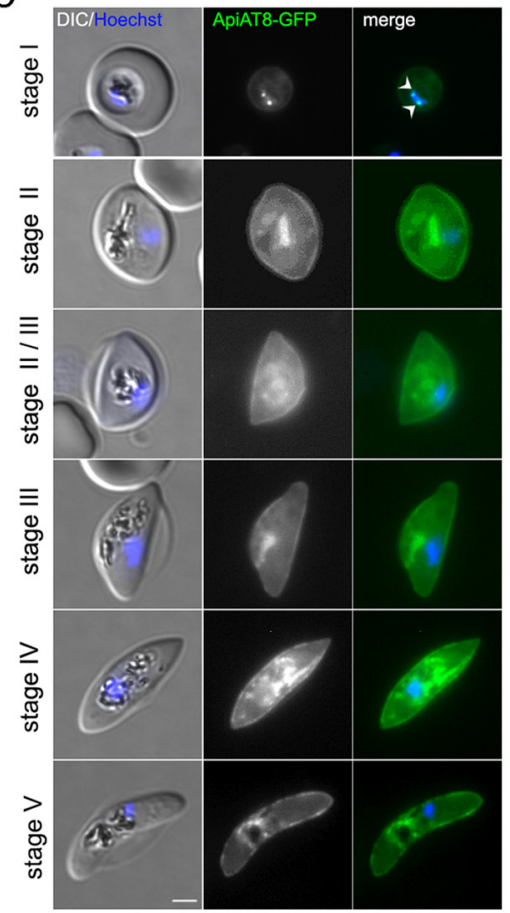

F

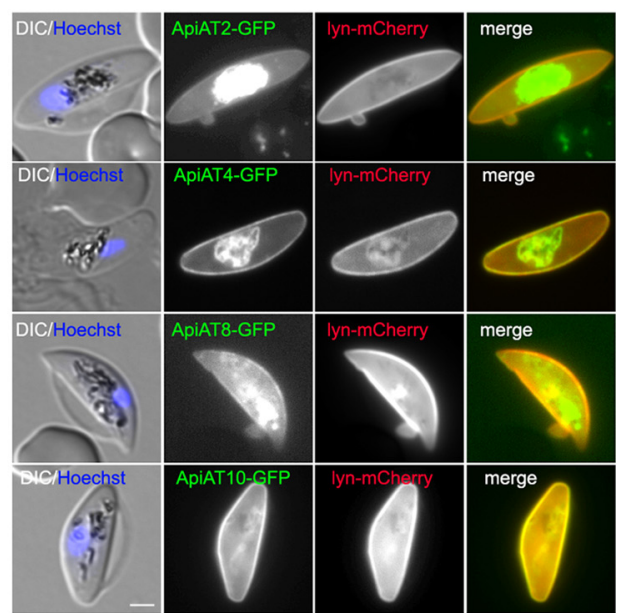

FIG 4 P. falciparum ApiATs localize to the parasite plasma membrane (PPM) during gametocyte development. (A to D) Localization of PfApiAT2-GFP (A), PfApiAT4-GFP (B), PfApiAT8-GFP (C), and PfApiAT10-GFP (D) in individual 3D7-iGP-ApiAT-GFP cell lines during gametocyte development (stages I to V) as determined by live-cell microscopy. White arrowheads indicate remaining GDV1-GFP signal observed in close proximity to the Hoechst signal in the 3D7iGP-ApiAT8-GFP cell line (C) as previously described $(60,83,84)$. (E) Localization of PfApiAT9-GFP during gametocytogenesis was assessed with the 3D7ApiAT9-GFP cell line (see Fig. 1) upon induction with choline depletion. (F) Colocalization of the GFP-tagged ApiAT fusion proteins with the PPM marker protein Lyn-mCherry. Nuclei were stained with Hoechst-33342. Bars, $2 \mu \mathrm{m}$. 
A
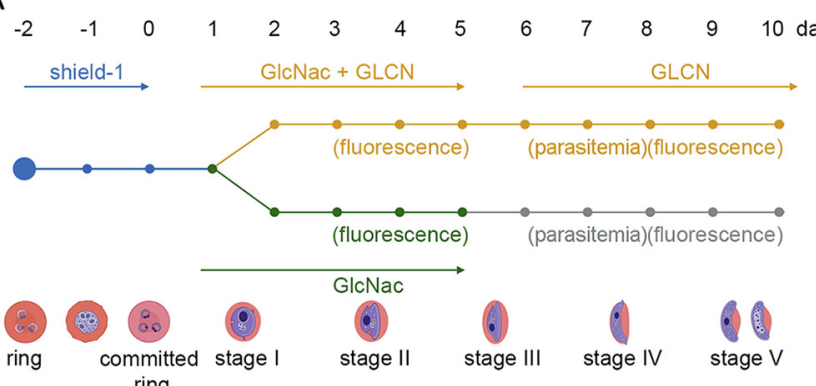

B
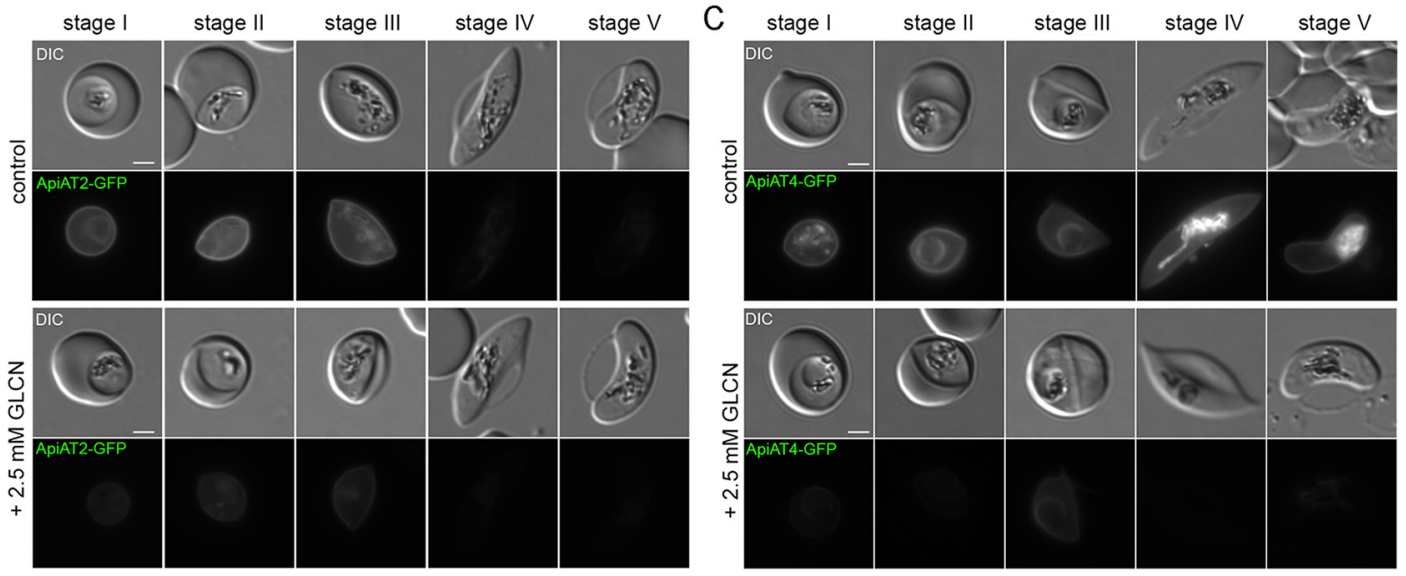

D

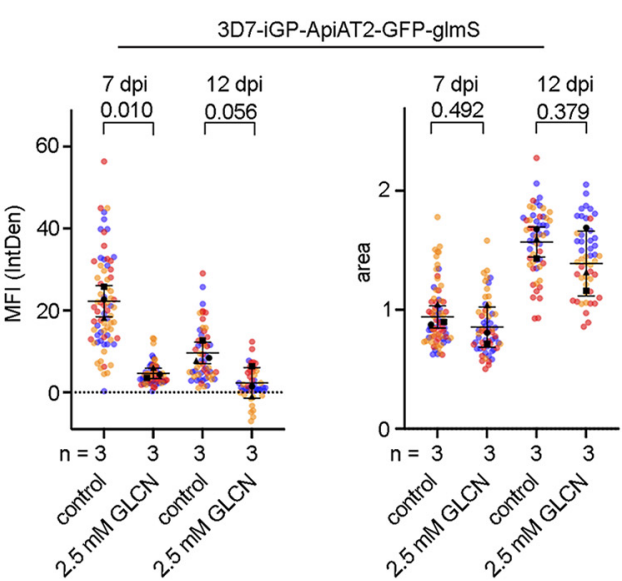

E

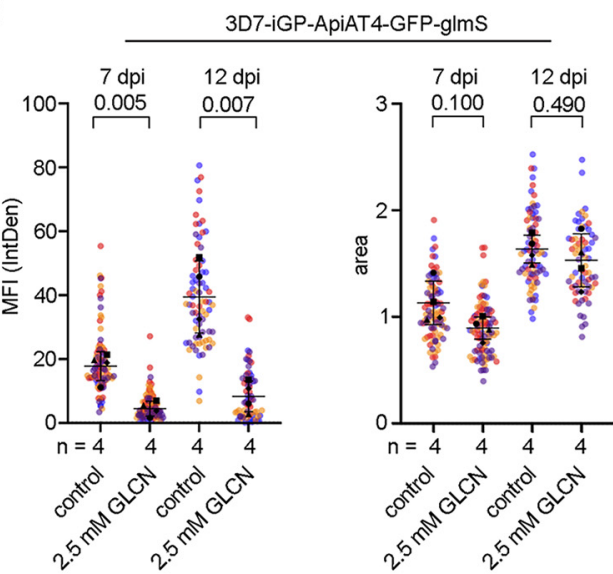

F

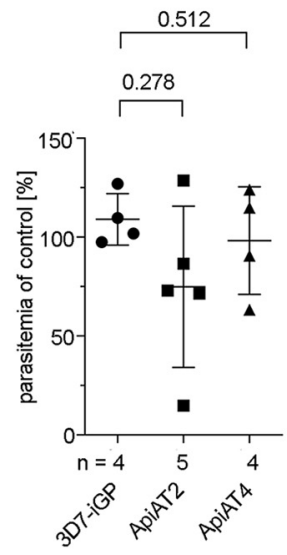

FIG 5 Conditional knockdown of PfApiAT2 and PfApiAT4 reveals dispensability for gametocyte development. (A) Schematic representation of the experimental setup. (B and C) Live-cell microscopy of parasites with identical settings of 3D7-iGP-ApiAT2-GFP

(Continued on next page) 


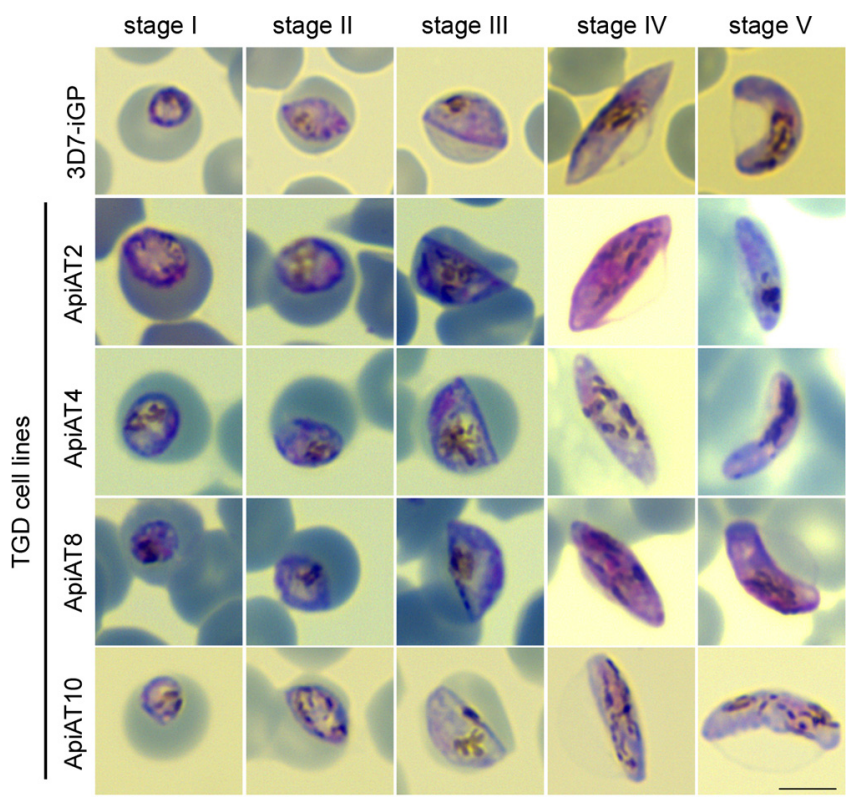

FIG 6 Targeted gene disruption (TGD) of PfApiAT2, PfApiAT4, PfApiAT8, and PfApiAT10 reveals dispensability of ApiATs for gametocyte development. Representative images from two (PfApiAT2 and PfApiAT4) or three (PfApiAT8 and PfApiAT10) independent experiments derived from Giemsa-stained thin blood smears of gametocyte stages I to $V$ of 3D7-iGP, 3D7-iGP-ApiAT2-TGD, 3D7-iGP-ApiAT4TGD, 3D7-iGP-ApiAT8-TGD, and 3D7-iGP-ApiAT10-TGD parasites. Bar, $5 \mu \mathrm{m}$.

Induction of gametocytogenesis was done as previously described (41, 60). Briefly, GDV1-GFP-DD expression was achieved by the addition of 2 or $4 \mu \mathrm{M}$ Shield-1 to the culture medium, and gametocyte cultures were treated with $50 \mathrm{mM} \mathrm{N}$-acetyl-D-glucosamine (GICNAc) for 5 days starting $72 \mathrm{~h}$ after Shield1 addition to eliminate asexual parasites (61). Alternatively, asexual ring stage cultures with $>10 \%$ parasitemia, cultured in the presence of choline, were synchronized with sorbitol (59) and washed twice in choline-free RPMI medium. Cells were kept in choline-free medium for the entirety of the assay. After one reinvasion cycle, cultures at trophozoite stage were treated with $50 \mathrm{mM}$ GlcNAc (61) and kept on this for 5 days. Gametocytes were maintained in RPMI complete medium containing $0.25 \%$ Albumax and $0.25 \%$ sterile filtered human serum (Interstate Blood Bank, Inc., Memphis, TN, USA).

Growth assays in low-amino acid medium were performed using amino acid-restricted RPMI medium prepared as previously described (20). Briefly, complete medium was added in a 1/20 dilution to amino acid-free RPMI 1640 medium (catalog no. R9010-01; US Biological). This resulted in a 1:20 of the concentration of every amino acid compared to the standard complete RPMI-based medium.

Cloning of plasmid constructs for parasite transfection. For endogenous tagging using the SLI system (38) and glmS-based conditional knockdown (40), a 855-bp (PfApiAT4/MFR5/PF3D7_1129900) and 1,001-bp (PfApiAT2/MFR4/PF3D7_0914700) homology region was amplified using 3D7 guide DNA (gDNA) and cloned into pSLI-PIC1-GFP-glmS (62) using the Notl and Mlul restriction sites.

For endogenous GFP tagging, a 886-bp (PfApiAT8/NPT1/PF3D7_0104800), 953-bp (PfApiAT9/MFR2/ PF3D7_0104700), and 881-bp (PfApiAT10/MFR3/PF3D7_0312500) homology region was amplified using 3D7 gDNA and cloned into pSLI-GFP (38) using the Notl and Mlul restriction sites.

For SLI-based targeted gene disruption (SLI-TGD), a 396-bp (PfApiAT4 and PfApiAT8), 402-bp (PfApiAT9), 393-bp (PfApiAT10), and 435-bp (PfApiAT2) homology region was amplified using 3D7 gDNA and cloned into the PSLI-TGD plasmid (38) using the Notl and Mlul restriction sites.

For overexpression constructs, the full-length PfApiAT10 sequence was amplified from parasite gDNA and cloned into PARL-ama1 (46)-AIP-mCherry-yDHODH (62) using the Xhol and Kpnl restriction

\section{FIG 5 Legend (Continued)}

(B) and 3D7-iGP-ApiAT4-GFP (C) stage I to V gametocytes. Bars, $2 \mu \mathrm{m}$. (D and E) Quantification of knockdown by measuring mean fluorescence intensity (MFI) density and size (area) of 3D7-iGP-ApiAT2-GFP (D) and 3D7-iGP-ApiAT4-GFP (E) parasites at day 7 and day 12 postinduction of gametocytogenesis cultured either with or without (control) $2.5 \mathrm{mM} \mathrm{GLCN}$. Data are displayed as means \pm SD of three (3D7-iGP-ApiAT2-GFP) or four (3D7-iGP-ApiAT4-GFP) independent experiments, and individual data points are displayed on a scatterplot color coded by experiments according to SuperPlots guidelines (82). $P$ values displayed were determined with a two-tailed unpaired $t$ test with Welch's correction. (F) For each condition, gametocytemia at day 10 postgametocyte induction was determined by counting between 702 and 7,693 (mean, 2,210) cells per condition in Giemsastained thin blood smears. The relative gametocytemia values (as a percentage) displayed were obtained by dividing the gametocytemia of glucosamine-treated cultures by the gametocytemia of the corresponding untreated cultures. Displayed are means \pm SD of independent growth experiments with the number of experiments $(n)$ indicated. A two-tailed unpaired $t$ test with Welch's and Benjamini-Hochberg correction was used to calculate multiplicity-adjusted $P$ values for ApiAT2-GFPglmS or ApiAT4-GFP-glmS versus 3D7-iGP parasites all cultured with $2.5 \mathrm{mM} \mathrm{GLCN}$. 
sites or into the PARL-crt (45)-PF3D7_0324600-GFP-hDHFR (37) plasmid using the Kpnl and Avrll restriction sites. Oligonucleotides used to generate the DNA fragments are summarized in Table S1 in the supplemental material.

For colocalization experiments, the plasmid pLyn-FRB-mCherry (38) was used.

Western blot analysis. Immunoblots were performed using saponin-lysed, infected erythrocytes. Parasite proteins were separated on a $12 \%$ sodium dodecyl sulfate-polyacrylamide gel (SDS-PAG) as described previously $(63,64)$ and transferred to a nitrocellulose membrane (Amersham Protran; 0.45$\mu \mathrm{m}$-pore-size nitrocellulose membrane; GE Healthcare) using a Trans-Blot device (Bio-Rad) according to the manufacturer's instructions. The membranes were blocked with $3 \%$ skim milk in Tris-buffered saline (TBS) for $30 \mathrm{~min}$ and then probed with mouse anti-GFP (clone 7.1 and 13.1; 1:1,000, Roche) or rabbit anti-aldolase $(65)(1: 2,000)$. The chemiluminescent signal of the horseradish peroxidase-coupled secondary antibodies (Dianova) was visualized using a Chemi Doc XRS imaging system (Bio-Rad) and processed with Image Lab 5.2 software (Bio-Rad).

To perform loading controls and ensure equal loading of parasite material, rabbit antialdolase (65) antibodies were used. The corresponding immunoblots were incubated twice in stripping buffer $(0.2 \mathrm{M}$ glycine, $50 \mathrm{mM}$ dithiothreitol, $0.05 \%$ Tween 20 ) at $55^{\circ} \mathrm{C}$ for $1 \mathrm{~h}$ and washed three times with Tris-buffered saline for $10 \mathrm{~min}$ before reprobing.

Transfection of $\boldsymbol{P}$. falciparum. For transfection, Percoll-purified (58) parasites at late schizont stage were transfected with $50 \mu \mathrm{g}$ plasmid DNA using Amaxa Nucleofector 2b (Lonza, Switzerland) as previously described (66). Transfectants were selected using either 4 nM WR99210 (Jacobus Pharmaceuticals), $0.9 \mu \mathrm{M}$ DSM1 (67) (BEl Resources), or $2 \mu \mathrm{g} / \mathrm{ml}$ blasticidin S (Life Technologies, USA). In order to select for parasites carrying the genomic modification via the SLI system (38), G418 (ThermoFisher, USA) at a final concentration of $400 \mu \mathrm{g} / \mathrm{ml}$ was added to a culture with about $5 \%$ parasitemia. The selection process and integration test were performed as previously described (38).

Imaging. All fluorescence images were captured using a Zeiss Axioskop 2plus microscope with a Hamamatsu digital camera (model C4742-95) or a Leica D6B fluorescence microscope equipped with a Leica DFC9000 GT camera and a Leica Plan Apochromat $100 \times / 1.4$ oil objective.

Microscopy of live-parasite-infected erythrocytes was performed as previously described (68). Briefly, parasites were incubated in standard culture medium with $1 \mu \mathrm{g} / \mathrm{ml}$ Hoechst-33342 (Invitrogen) for 15 min at $37^{\circ} \mathrm{C}$ prior to imaging. Infected erythrocytes $(5.4 \mu \mathrm{l})$ were added on a glass slide and covered with a cover slip. Nuclei were stained with $1 \mu \mathrm{g} / \mathrm{ml}$ Hoechst-33342 (Invitrogen). Mitochondria were visualized by incubation of parasites with $20 \mathrm{nM}$ MitoTracker Red 665 CMXRos (Invitrogen) for $15 \mathrm{~min}$ at $37^{\circ} \mathrm{C}$ prior to imaging. Contrast and intensities were linear adjusted for clarification and cropped images were assembled as panels using Fiji (69) and Adobe Photoshop CC 2021.

Growth assay. For growth assays of TGD cell lines, a flow cytometry assay, adapted from previously published assays $(70,71)$, was performed to measure proliferation over 5 days. For growth under lowamino acid conditions, TGD and wild-type cell lines were cultured in parallel in standard and amino acid-depleted medium for 5 days. Each day parasite cultures were resuspended, and $20-\mu l$ samples were transferred to an Eppendorf tube. Eighty microliters of RPMI medium containing Hoechst-33342 and dihydroethidium (DHE) was added to obtain final concentrations of $5 \mu \mathrm{g} / \mathrm{ml}$ and $4.5 \mu \mathrm{g} / \mathrm{ml}$, respectively. Samples were incubated for $20 \mathrm{~min}$ (protected from UV light) at room temperature, and parasitemia was determined using an LSRII flow cytometer by counting 100,000 events using the FACSDiva software (BD Biosciences) or using an ACEA NovoCyte flow cytometer.

Gametocyte quantification assay. Giemsa-stained blood smears at day 10 postinduction of GDV1 expression were obtained, and at least 10 fields of view were recorded using a $63 \times$ objective per treatment and time point. Erythrocyte numbers were then determined using the automated Parasitemia software (http://www.gburri.org/parasitemia/), while the number of gametocytes was determined manually in $>700$ erythrocytes per sample.

gImS-based knockdown. The glmS-based knockdown assay was adapted from previously published assays $(40,72)$. To induce knockdown, highly synchronous early ring stage parasites were split in two dishes, $2.5 \mathrm{mM}$ glucosamine was added to one of them, and parasite growth was measured by fluorescence-activated cell sorting (FACS) after two and four parasite replication cycles. Parasite cultures were inspected daily by Giemsa smears and, if necessary, diluted to avoid growth bias caused by high parasitemia. As an additional control, the same amount of glucosamine was also added to 3D7 wild-type parasites. For all analyses, medium was changed daily, and fresh glucosamine was added every day.

Knockdown was quantified by fluorescence live-cell microscopy using schizonts about $40 \mathrm{~h}$ after glucosamine treatment. Parasites of similar sizes were imaged, and fluorescence was captured with the same acquisition settings to obtain comparable measurements of the fluorescence intensity. Fluorescence intensity (integrated density) was measured with Fiji (69), and background was subtracted in each image. The data were visualized with GraphPad Prism version 8 (GraphPad Software, USA).

For knockdown experiments in gametocytes, synchronized ring stage cultures were induced by the addition of Shield-1 as described above. At day 3 postinduction, the culture was spilt into two dishes, and one dish was cultured in the presence of $2.5 \mathrm{mM}$ glucosamine for the remaining 10 days. Knockdown was quantified by fluorescence live-cell microscopy at day 7 and 10 postinduction as described above.

Drug assays. Drug assays were adapted from previously described assays (73-75). Briefly, 3D7-iGP and 3D7-iGP-ApiAT10-TGD parasites were synchronized to a 4-h time window resulting in 0- to 4-h ring stage parasites. At $24 \mathrm{hpi}$, parasitemia was determined by flow cytometry, and the drug susceptibility assays were set up in black 96 -well microtiter plates (Thermo Scientific) with $0.1 \%$ starting parasitemia and $2 \%$ hematocrit in a final volume of $200 \mu \mathrm{l}$. In each plate, infected erythrocytes in the absence of drugs treated with dimethyl sulfoxide (DMSO) only served as positive controls, while uninfected red 
blood cells (RBCs) served as negative controls (for background subtraction). Parasites were incubated with various concentrations of dihydroartemisinin (DHA) (catalog no. AG-CN2-0468; Adipogen, Switzerland) (0 to $50 \mathrm{nM}$ ) and atovaquone (catalog no. 23802; Cayman) (0 to $16 \mathrm{NM}$ ).

After $96 \mathrm{~h}$ of incubation, parasite growth was determined by measuring the fluorescence of SYBR Gold (Invitrogen). Therefore, $100 \mu \mathrm{l} /$ well supernatant was discarded without disturbing the RBC layer and $100 \mu$ l of lysis buffer ( $20 \mathrm{mM}$ Tris, $0.008 \%$ saponin, $0.08 \%$ Triton X-100, $1 \times$ SYBR Gold) was added to each well. Plates were incubated in the dark for $2 \mathrm{~h}$ at room temperature before measuring fluorescence using the EnVision Multimode plate reader (PerkinElmer) as described previously (75). In order to calculate $50 \%$ infective concentration $\left(\mathrm{IC}_{50}\right)$ values, the measured values were normalized to the uninfected erythrocytes and plotted in GraphPad Prism version 8 (GraphPad Software, USA) as a percentage of the value for the DMSO control. Dose-response curves were generated using nonlinear regression (curve fit $>$ dose-response inhibition $>$ (log) inhibitor versus normalized response-variable slope).

Quantitative real-time PCR. Parasites at different time points (8, 16, 32, and $44 \mathrm{hpi})$ were harvested for 3D7-ApiAT2-TGD, 3D7-ApiAT4-TGD, 3D7-ApiAT8-TGD, 3D7-ApiAT10-TGD, and 3D7-WT to obtain RNA samples for quantitative real-time PCR (qPCR). Highly synchronous ring stage parasite cultures were grown for another $40 \mathrm{~h}$, and TRIzol samples were harvested in the following cycle. Volumes of prewarmed TRIzol used for infected erythrocyte lysis and storage of RNA samples depended on the parasite stage: ring stages were lysed in $5 \times$ volumes, trophozoites in $10 \times$ volumes, and schizonts in $20 \times$ volumes of the settled cell pellet. RNA was purified and checked for the absence of genomic DNA. cDNA synthesis with random hexamers, and quantitative real-time PCR was performed exactly as previously described (76). Primers for each of the apiat genes, for genes to control for parasite stages (sbp 1 [77], tom22, and ama1) and for housekeeping genes (arginyl-tRNA synthetase [76] and fructose-bisphosphate aldolase [78] genes) are listed in Table S1. Amplification efficiencies of the primer pairs were determined over $6 \log _{10}$ dilutions of gDNA (10 $\mathrm{ng}$ to $0.0001 \mathrm{ng}$ ) and were shown to have similar values between 1.915 and 2.001 (Table S1). Expression of apiat genes and controls were analyzed in relation to expression of the arginyl-tRNA synthetase gene (normalizer).

Software. Schematic protein representations were designed using IBS (79), predicted protein domains were obtained from plasmodDB (80) inferred from TMHMM (81). Parasite icons were generated using BioRender (biorender.com). Statistical analyses were performed with GraphPad Prism version 8 (GraphPad Software, USA).

\section{SUPPLEMENTAL MATERIAL}

Supplemental material is available online only.

FIG S1, TIF file, 0.9 MB.

FIG S2, TIF file, $0.9 \mathrm{MB}$.

FIG S3, TIF file, $0.5 \mathrm{MB}$.

FIG S4, JPG file, 1 MB.

FIG S5, TIF file, 1.6 MB.

TABLE S1, PDF file, $0.03 \mathrm{MB}$.

\section{ACKNOWLEDGMENTS}

We thank Till Voss for 3D7-iGP parasites, Jacobus Pharmaceuticals for WR99210, Greg Burri for the parasitemia software, and Eva Lorenz for statistical support. DSM1 (MRA-1161) was obtained from MR4/BEl Resources, NIAID, NIH.

A.B. and J.S.W. were funded by the German Research Foundation (DFG) grant BA 5213/3-1. This project was also supported by Partnership of Universität Hamburg and DESY (PIER) project identifier (ID) PIF-2018-87 and by a Joachim Herz Foundation Project grant. J.L.F. is supported by a HFSPO long-term postdoctoral fellowship (LT000024/2020-L). P.-C.B. was funded by the Deutsche Forschungsgemeinschaft (DFG, German Research Foundation) project 414222880.

Conceptualization, J.S.W., J.S., T.-W.G., and A.B.; methodology, J.S.W., E.P., P.-C.B., P.M.-R., T.S., J.S., T.-W.G., and A.B.; investigation, J.S.W., C.V.G., G.F., J.M.R., E.P., J.L.F., H.V.T., S.S., and P.-C.B.; formal analysis, J.S. and A.B.; writing original manuscript, J.S.W. and A.B.; review and editing, J.S.W., T.-W.G., and A.B.; funding acquisition, T.-W.G., A.B., and J.S.; resources, P.M.-R. and T.S.; project administration, T.-W.G. and A.B.; supervision, T.-W.G. and A.B. All authors read and approved the manuscript.

\section{REFERENCES}

1. Krishnan A, Soldati-Favre D. 2021. Amino acid metabolism in apicomplexan parasites. Metabolites 11:61. https://doi.org/10.3390/metabo11020061.

2. Wrenger C, Müller IB, Schifferdecker AJ, Jain R, Jordanova R, Groves MR. 2011. Specific inhibition of the aspartate aminotransferase of Plasmodium falciparum. J Mol Biol 405:956-971. https://doi.org/10.1016/j.jmb.2010.11 .018 .

3. Nagaraj VA, Mukhi D, Sathishkumar V, Subramani PA, Ghosh SK, Pandey RR, Shetty MC, Padmanaban G. 2015. Asparagine requirement in 
Plasmodium berghei as a target to prevent malaria transmission and liver infections. Nat Commun 6:8775. https://doi.org/10.1038/ncomms9775.

4. Meireles P, Mendes AM, Aroeira RI, Mounce BC, Vignuzzi M, Staines HM, Prudêncio M. 2017. Uptake and metabolism of arginine impact Plasmodium development in the liver. Sci Rep 7:4072. https://doi.org/10.1038/ s41598-017-04424-y.

5. MacRae Jl, Sheiner L, Nahid A, Tonkin C, Striepen B, McConville MJ. 2012. Mitochondrial metabolism of glucose and glutamine is required for intracellular growth of Toxoplasma gondii. Cell Host Microbe 12:682-692. https://doi.org/10.1016/j.chom.2012.09.013.

6. Sopitthummakhun K, Thongpanchang C, Vilaivan T, Yuthavong Y, Chaiyen $P$, Leartsakulpanich U. 2012. Plasmodium serine hydroxymethyltransferase as a potential anti-malarial target: inhibition studies using improved methods for enzyme production and assay. Malar J 11:194. https://doi.org/10 .1186/1475-2875-11-194.

7. Spielmann T, Gras S, Sabitzki R, Meissner M. 2020. Endocytosis in Plasmodium and Toxoplasma parasites. Trends Parasitol 36:520-532. https://doi .org/10.1016/j.pt.2020.03.010.

8. Kirk K, Lehane AM. 2014. Membrane transport in the malaria parasite and its host erythrocyte. Biochem J 457:1-18. https://doi.org/10.1042/BJ20131007.

9. Divo AA, Geary TG, Davis NL, Jensen JB. 1985. Nutritional requirements of Plasmodium falciparum in culture. I. Exogenously supplied dialyzable components necessary for continuous growth. J Protozool 32:59-64. https://doi.org/10.1111/j.1550-7408.1985.tb03013.x.

10. Ginsburg H, Kutner S, Krugliak M, Cabantchik Zl. 1985. Characterization of permeation pathways appearing in the host membrane of Plasmodium falciparum infected red blood cells. Mol Biochem Parasitol 14:313-322. https://doi.org/10.1016/0166-6851(85)90059-3.

11. Kirk K, Horner HA, Elford BC, Ellory JC, Newbold Cl. 1994. Transport of diverse substrates into malaria-infected erythrocytes via a pathway showing functional characteristics of a chloride channel. J Biol Chem 269: 3339-3347. https://doi.org/10.1016/S0021-9258(17)41868-0.

12. Olszewski KL, Morrisey JM, Wilinski D, Burns JM, Vaidya AB, Rabinowitz JD, Llinás M. 2009. Host-parasite interactions revealed by Plasmodium falciparum metabolomics. Cell Host Microbe 5:191-199. https://doi.org/10 .1016/j.chom.2009.01.004.

13. Liu J, Istvan ES, Gluzman IY, Gross J, Goldberg DE. 2006. Plasmodium falciparum ensures its amino acid supply with multiple acquisition pathways and redundant proteolytic enzyme systems. Proc Natl Acad Sci U S A 103:8840-8845. https://doi.org/10.1073/pnas.0601876103.

14. Babbitt SE, Altenhofen L, Cobbold SA, Istvan ES, Fennell C, Doerig C, Llinás $M$, Goldberg DE. 2012. Plasmodium falciparum responds to amino acid starvation by entering into a hibernatory state. Proc Natl Acad Sci U S A 109:E3278-E3287. https://doi.org/10.1073/pnas.1209823109.

15. McLean KJ, Jacobs-Lorena M. 2020. The response of Plasmodium falciparum to isoleucine withdrawal is dependent on the stage of progression through the intraerythrocytic cell cycle. Malar J 19:147. https://doi.org/10 1186/s12936-020-03220-w.

16. Martin RE, Kirk K. 2007. Transport of the essential nutrient isoleucine in human erythrocytes infected with the malaria parasite Plasmodium falciparum. Blood 109:2217-2224. https://doi.org/10.1182/blood-2005-11-026963.

17. Cobbold SA, Martin RE, Kirk K. 2011. Methionine transport in the malaria parasite Plasmodium falciparum. Int J Parasitol 41:125-135. https://doi .org/10.1016/j.ijpara.2010.09.001.

18. Desai SA, Krogstad DJ, McCleskey EW. 1993. A nutrient-permeable channel on the intraerythrocytic malaria parasite. Nature 362:643-646. https:// doi.org/10.1038/362643a0.

19. Desai SA, Rosenberg RL. 1997. Pore size of the malaria parasite's nutrient channel. Proc Natl Acad Sci U S A 94:2045-2049. https://doi.org/10.1073/ pnas.94.5.2045.

20. Mesén-Ramírez P, Bergmann B, Tran TT, Garten M, Stäcker J, Naranjo-Prado I, Höhn K, Zimmerberg J, Spielmann T. 2019. EXP1 is critical for nutrient uptake across the parasitophorous vacuole membrane of malaria parasites. PLoS Biol 17:e3000473. https://doi.org/10.1371/journal.pbio.3000473.

21. Garten M. 2018. EXP2 is a nutrient-permeable channel in the vacuolar membrane of Plasmodium and is essential for protein export via PTEX. Nat Microbiol 3:1090-1098. https://doi.org/10.1038/s41564-018-0222-7.

22. Counihan NA, Modak JK, de Koning-Ward TF. 2021. How malaria parasites acquire nutrients from their host. Front Cell Dev Biol 9:649184. https://doi .org/10.3389/fcell.2021.649184.

23. Martin RE. 2020. The transportome of the malaria parasite. Biol Rev Camb Philos Soc 95:305-332. https://doi.org/10.1111/brv.12565.

24. Martin RE, Henry RI, Abbey JL, Clements JD, Kirk K. 2005. The 'permeome' of the malaria parasite: an overview of the membrane transport proteins of Plasmodium falciparum. Genome Biol 6:R26. https://doi.org/10.1186/ gb-2005-6-3-r26.

25. Parker KER, Fairweather SJ, Rajendran E, Blume M, McConville MJ, Bröer S Kirk K, van Dooren GG. 2019. The tyrosine transporter of Toxoplasma gondii is a member of the newly defined apicomplexan amino acid transporter (ApiAT) family. PLoS Pathog 15:e1007577. https://doi.org/10.1371/ journal.ppat.1007577.

26. Rajendran E, Clark M, Goulart C, Steinhöfel B, Tjhin ET, Gross S, Smith NC, Kirk K, van Dooren GG. 2021. Substrate-mediated regulation of the arginine transporter of Toxoplasma gondii. PLoS Pathog 17:e1009816. https://doi.org/10.1371/journal.ppat.1009816.

27. Reddy VS, Shlykov MA, Castillo R, Sun El, Saier MH. 2012. The major facilitator superfamily (MFS) revisited. FEBS J 279:2022-2035. https://doi.org/ 10.1111/j.1742-4658.2012.08588.x.

28. Pao SS, Paulsen IT, Saier MH. 1998. Major facilitator superfamily. Microbiol Mol Biol Rev 62:1-34. https://doi.org/10.1128/MMBR.62.1.1-34.1998.

29. Rajendran E, Hapuarachchi SV, Miller CM, Fairweather SJ, Cai Y, Smith NC, Cockburn IA, Bröer S, Kirk K, van Dooren GG. 2017. Cationic amino acid transporters play key roles in the survival and transmission of apicomplexan parasites. Nat Commun 8:14455. https://doi.org/10.1038/ncomms14455.

30. Boisson B, Lacroix C, Bischoff E, Gueirard P, Bargieri DY, Franke-Fayard B, Janse CJ, Ménard R, Baldacci P. 2011. The novel putative transporter NPT1 plays a critical role in early stages of Plasmodium berghei sexual development. Mol Microbiol 81:1343-1357. https://doi.org/10.1111/j.1365-2958 2011.07767.x.

31. Blomqvist K, Helmel M, Wang C, Absalon S, Labunska T, Rudlaff RM Adapa S, Jiang R, Steen H, Dvorin JD. 2020. Influence of Plasmodium falciparum calcium-dependent protein kinase 5 (PfCDPK5) on the late schizont stage phosphoproteome. mSphere 5:e00921-19. https://doi .org/10.1128/mSphere.00921-19.

32. Kloehn J, Lunghi M, Varesio E, Dubois D, Soldati-Favre D. 2021. Untargeted metabolomics uncovers the essential lysine transporter in Toxoplasma gondii. Metabolites 11:476. https://doi.org/10.3390/metabo11080476.

33. Wallbank BA, Dominicus CS, Broncel M, Legrave N, Kelly G, MacRae ال Staines HM, Treeck M. 2019. Characterisation of the Toxoplasma gondii tyrosine transporter and its phosphorylation by the calcium-dependent protein kinase 3. Mol Microbiol 111:1167-1181. https://doi.org/10.1111/ mmi.14156.

34. Fairweather SJ, Rajendran E, Blume M, Javed K, Steinhöfel B, McConville MJ, Kirk K, Bröer S, van Dooren GG. 2021. Coordinated action of multiple transporters in the acquisition of essential cationic amino acids by the intracellular parasite Toxoplasma gondii. PLoS Pathog 17:e1009835. https://doi.org/ 10.1371/journal.ppat.1009835.

35. Kenthirapalan S, Waters AP, Matuschewski K, Kooij TWA. 2016. Functional profiles of orphan membrane transporters in the life cycle of the malaria parasite. Nat Commun 7:10519. https://doi.org/10.1038/ncomms10519.

36. Rocamora F, Gupta $\mathrm{P}$, Istvan ES, Luth MR, Carpenter EF, Kümpornsin K Sasaki E, Calla J, Mittal N, Carolino K, Owen E, Llinás M, Ottilie S, Goldberg DE, Lee MCS, Winzeler EA. 2021. PfMFR3: a multidrug-resistant modulator in Plasmodium falciparum. ACS Infect Dis 7:811-825. https://doi.org/10 .1021 /acsinfecdis.0c00676

37. Wichers JS, Scholz JAM, Strauss J, Witt S, Lill A, Ehnold L-I, Neupert $\mathrm{N}_{\text {, }}$ Liffner B, Lühken R, Petter M, Lorenzen S, Wilson DW, Löw C, Lavazec C, Bruchhaus I, Tannich E, Gilberger TW, Bachmann A. 2019. Dissecting the gene expression, localization, membrane topology, and function of the Plasmodium falciparum STEVOR protein family. mBio 10:e01500-19. https://doi.org/10.1128/mBio.01500-19.

38. Birnbaum J, Flemming $S$, Reichard $N$, Soares $A B$, Mesén-Ramírez $P$, Jonscher E, Bergmann B, Spielmann T. 2017. A genetic system to study Plasmodium falciparum protein function. Nat Methods 14:450-456. https://doi.org/10 $.1038 /$ nmeth.4223.

39. Inoue T, Heo W, Grimley JS, Wandless TJ, Meyer T. 2005. An inducible trans location strategy to rapidly activate and inhibit small GTPase signaling pathways. Nat Methods 2:415-418. https://doi.org/10.1038/nmeth763.

40. Prommana $P$, Uthaipibull C, Wongsombat C, Kamchonwongpaisan S, Yuthavong Y, Knuepfer E, Holder AA, Shaw PJ. 2013. Inducible knockdown of Plasmodium gene expression using the glmS ribozyme. PLoS One 8:e73783. https://doi.org/10.1371/journal.pone.0073783.

41. Boltryk SD, Passecker A, Alder A, Carrington $E$, van de Vegte-Bolmer $M$, van Gemert G-J, van der Starre A, Beck H-P, Sauerwein RW, Kooij TWA Brancucci NMB, Proellochs NI, Gilberger T-W, Voss TS. 2021. CRISPR/Cas9engineered inducible gametocyte producer lines as a valuable tool for Plasmodium falciparum malaria transmission research. Nat Commun 12: 4806. https://doi.org/10.1038/s41467-021-24954-4 
42. Otto TD, Wilinski D, Assefa S, Keane TM, Sarry LR, Böhme U, Lemieux J, Barrell B, Pain A, Berriman M, Newbold C, Llinás M. 2010. New insights into the blood-stage transcriptome of Plasmodium falciparum using RNA-Seq. Mol Microbiol 76:12-24. https://doi.org/10.1111/j.1365-2958 2009.07026.x

43. López-Barragán MJ, Lemieux J, Quiñones M, Williamson KC, Molina-Cruz A, Cui K, Barillas-Mury C, Zhao K, Su X. 2011. Directional gene expression and antisense transcripts in sexual and asexual stages of Plasmodium falciparum. BMC Genomics 12:587. https://doi.org/10.1186/1471-2164-12-587.

44. Sangaré LO, Alayi TD, Westermann B, Hovasse A, Sindikubwabo F, Callebaut I, Werkmeister E, Lafont F, Slomianny C, Hakimi MA, Van Dorsselaer A, Schaeffer-Reiss C, Tomavo S. 2016. Unconventional endosome-like compartment and retromer complex in Toxoplasma gondii govern parasite integrity and host infection. Nat Commun 7:11191. https://doi.org/10.1038/ ncomms11191.

45. Struck NS, de Souza Dias S, Langer C, Marti M, Pearce JA, Cowman AF, Gilberger TW. 2005. Re-defining the Golgi complex in Plasmodium falciparum using the novel Golgi marker PfGRASP. J Cell Sci 118:5603-5613. https://doi.org/10.1242/jcs.02673.

46. Hu G, Cabrera A, Kono M, Mok S, Chaal BK, Haase S, Engelberg K, Cheemadan S, Spielmann T, Preiser PR, Gilberger T-W, Bozdech Z. 2010. Transcriptional profiling of growth perturbations of the human malaria parasite Plasmodium falciparum. Nat Biotechnol 28:91-98. https://doi .org/10.1038/nbt.1597.

47. Hudson AT, Randall AW, Fry M, Ginger CD, Hill B, Latter VS, McHardy N, Williams RB. 1985. Novel anti-malarial hydroxynaphthoquinones with potent broad spectrum anti-protozoal activity. Parasitology 90:45-55. https://doi.org/10.1017/S0031182000049003.

48. Wellems T, Oduola AM, Fenton B, Desjardins R, Panton L. 1988. Chromosome size variation occurs in cloned Plasmodium falciparum on in vitro cultivation. Rev Bras Genét 11:813-825.

49. Otto TD, Böhme U, Sanders M, Reid A, Bruske El, Duffy CW, Bull PC, Pearson RD, Abdi A, Dimonte S, Stewart LB, Campino S, Kekre M, Hamilton WL, Claessens A, Volkman SK, Ndiaye D, Amambua-Ngwa A, Diakite M, Fairhurst RM, Conway DJ, Franck M, Newbold Cl, Berriman M. 2018. Long read assemblies of geographically dispersed Plasmodium falciparum isolates reveal highly structured subtelomeres. Wellcome Open Res 3:52. https://doi.org/10.12688/wellcomeopenres.14571.1.

50. van Esveld SL, Meerstein-Kessel L, Boshoven C, Baaij JF, Barylyuk K, Coolen JPM, van Strien J, Duim RAJ, Dutilh BE, Garza DR, Letterie M, Proellochs NI, de Ridder MN, Venkatasubramanian PB, de Vries LE, Waller RF, Kooij TWA, Huynen MA. 2021. A prioritized and validated resource of mitochondrial proteins in Plasmodium identifies unique biology. mSphere 6:e00614-21. https://doi.org/10.1128/mSphere.00614-21.

51. Goldberg DE. 2013. Complex nature of malaria parasite hemoglobin degradation. Proc Natl Acad Sci U S A 110:5283-5284. https://doi.org/10 .1073/pnas.1303299110.

52. Payne SH, Loomis WF. 2006. Retention and loss of amino acid biosynthetic pathways based on analysis of whole-genome sequences. Eukaryot Cell 5:272-276. https://doi.org/10.1128/EC.5.2.272-276.2006.

53. Sayers CP, Mollard V, Buchanan HD, McFadden GI, Goodman CD. 2018. A genetic screen in rodent malaria parasites identifies five new apicoplast putative membrane transporters, one of which is essential in human malaria parasites. Cell Microbiol 20:e12789. https://doi.org/10.1111/cmi .12789.

54. Ngotho P, Soares AB, Hentzschel F, Achcar F, Bertuccini L, Marti M. 2019. Revisiting gametocyte biology in malaria parasites. FEMS Microbiol Rev 43:401-414. https://doi.org/10.1093/femsre/fuz010.

55. Stanway RR, Bushell E, Chiappino-Pepe A, Roques M, Sanderson T, Franke-Fayard B, Caldelari R, Golomingi M, Nyonda M, Pandey V, Schwach F, Chevalley S, Ramesar J, Metcalf T, Herd C, Burda P-C, Rayner JC, SoldatiFavre D, Janse CJ, Hatzimanikatis V, Billker O, Heussler VT. 2019. Genomescale identification of essential metabolic processes for targeting the Plasmodium liver stage. Cell 179:1112-1128.e26. https://doi.org/10.1016/ j.cell.2019.10.030

56. Walliker D, Quakyi IA, Wellems TE, McCutchan TF, Szarfman A, London WT, Corcoran LM, Burkot TR, Carter R. 1987. Genetic analysis of the human malaria parasite Plasmodium falciparum. Science 236:1661-1666. https:// doi.org/10.1126/science.3299700.

57. Trager W, Jensen JB. 1997. Continuous culture of Plasmodium falciparum: its impact on malaria research. Int J Parasitol 27:989-1006. https://doi .org/10.1016/S0020-7519(97)00080-5.
58. Rivadeneira E, Wasserman M, Espinal C. 1983. Separation and concentration of schizonts of Plasmodium falciparum by Percoll gradients. J Protozool 30:367-370. https://doi.org/10.1111/j.1550-7408.1983.tb02932.x.

59. Lambros C, Vanderberg JP. 1979. Synchronization of Plasmodium falciparum erythrocytic stages in culture. J Parasitol 65:418-420. https://doi.org/ $10.2307 / 3280287$.

60. Filarsky M, Fraschka SA, Niederwieser I, Brancucci NMB, Carrington E, Carrió E, Moes S, Jenoe P, Bártfai R, Voss TS. 2018. GDV1 induces sexual commitment of malaria parasites by antagonizing HP1-dependent gene silencing. Science 359:1259-1263. https://doi.org/10.1126/science.aan6042.

61. Ponnudurai T, Lensen AHW, Meis JFGM, Meuwissen JHE. 1986. Synchronization of Plasmodium falciparum gametocytes using an automated suspension culture system. Parasitology 93:263-274. https://doi.org/10 $.1017 /$ S003118200005143X.

62. Wichers JS, Wunderlich J, Heincke D, Pazicky S, Strauss J, Schmitt M, Kimmel J, Wilcke L, Scharf S, von Thien H, Burda P-C, Spielmann T, Löw C, Filarsky M, Bachmann A, Gilberger TW. 2021. Identification of novel inner membrane complex and apical annuli proteins of the malaria parasite Plasmodium falciparum. Cell Microbiol 23:e13341. https://doi.org/10 $.1111 / \mathrm{cmi} .13341$

63. Bachmann A, Scholz JAM, Janßen M, Klinkert M-Q, Tannich E, Bruchhaus I, Petter M. 2015. A comparative study of the localization and membrane topology of members of the RIFIN, STEVOR and PfMC-2TM protein families in Plasmodium falciparum-infected erythrocytes. Malar J 14:274. https://doi.org/10.1186/s12936-015-0784-2.

64. Heiber A, Spielmann T. 2014. Preparation of parasite protein extracts and Western blot analysis. Bio-Protocol 4:e1136. https://doi.org/10.21769/ BioProtoc. 1136.

65. Mesén-Ramírez $P$, Reinsch F, Blancke Soares A, Bergmann B, Ullrich A-K, Tenzer S, Spielmann T. 2016. Stable translocation intermediates jam global protein export in Plasmodium falciparum parasites and link the PTEX component EXP2 with translocation activity. PLoS Pathog 12: e1005618. https://doi.org/10.1371/journal.ppat.1005618.

66. Moon RW, Hall J, Rangkuti F, Ho YS, Almond N, Mitchell GH, Pain A Holder AA, Blackman MJ. 2013. Adaptation of the genetically tractable malaria pathogen Plasmodium knowlesi to continuous culture in human erythrocytes. Proc Natl Acad Sci U S A 110:531-536. https://doi.org/10 .1073/pnas.1216457110.

67. Ganesan SM, Morrisey JM, Ke H, Painter HJ, Laroiya K, Phillips MA, Rathod PK, Mather MW, Vaidya AB. 2011. Yeast dihydroorotate dehydrogenase as a new selectable marker for Plasmodium falciparum transfection. Mol Biochem Parasitol 177:29-34. https://doi.org/10.1016/j.molbiopara.2011.01.004.

68. Grüring C, Spielmann T. 2012. Imaging of live malaria blood stage parasites. Methods Enzymol 506:81-92. https://doi.org/10.1016/B978-0-12-391856-7 .00029-9.

69. Schindelin J, Arganda-Carreras I, Frise E, Kaynig V, Longair M, Pietzsch T, Preibisch S, Rueden C, Saalfeld S, Schmid B, Tinevez J-Y, White DJ, Hartenstein V, Eliceiri K, Tomancak P, Cardona A. 2012. Fiji: an opensource platform for biological-image analysis. Nat Methods 9:676-682. https://doi.org/10.1038/nmeth.2019.

70. Jonscher E, Flemming S, Schmitt M, Sabitzki R, Reichard N, Birnbaum J, Bergmann B, Höhn K, Spielmann T. 2019. PfVPS45 is required for host cell cytosol uptake by malaria blood stage parasites. Cell Host Microbe 25: 166-173.e5. https://doi.org/10.1016/j.chom.2018.11.010.

71. Malleret B, Claser C, Ong ASM, Suwanarusk R, Sriprawat K, Howland SW, Russell B, Nosten F, Rénia L. 2011. A rapid and robust tri-color flow cytometry assay for monitoring malaria parasite development. Sci Rep 1:118. https://doi.org/10.1038/srep00118.

72. Burda P-C, Crosskey T, Lauk K, Zurborg A, Söhnchen C, Liffner B, Wilcke L, Pietsch E, Strauss J, Jeffries CM, Svergun DI, Wilson DW, Wilmanns M, Gilberger T-W. 2020. Structure-based identification and functional characterization of a lipocalin in the malaria parasite Plasmodium falciparum. Cell Rep 31:107817. https://doi.org/10.1016/j.celrep.2020.107817.

73. Burda P-C, Ramaprasad A, Pietsch E, Bielfeld S, Söhnchen C, Wilcke L, Strauss J, Schwudke D, Sait A, Collinson LM, Blackman MJ, Gilberger T-W. 2021. Global analysis of putative phospholipases in the malaria parasite Plasmodium falciparum reveals critical factors for parasite proliferation. bioRxiv https://doi.org/10.1101/2021.06.28.450158.

74. Smilkstein M, Sriwilaijaroen N, Kelly JX, Wilairat P, Riscoe M. 2004. Simple and Inexpensive fluorescence-based technique for high-throughput antimalarial drug screening. Antimicrob Agents Chemother 48:1803-1806. https://doi.org/10.1128/AAC.48.5.1803-1806.2004. 
75. Schwed SI, Alder A, Gilberger T, Kunick C. 2020. 4-Arylthieno[2,3-b]pyridine-2-carboxamides are a new class of antiplasmodial agents. Molecules 25:3187. https://doi.org/10.3390/molecules25143187.

76. Bachmann A, Bruske E, Krumkamp R, Turner L, Wichers JS, Petter M, Held J, Duffy MF, Sim BKL, Hoffman SL, Kremsner PG, Lell B, Lavstsen T, Frank M, Mordmüller B, Tannich E. 2019. Controlled human malaria infection with Plasmodium falciparum demonstrates impact of naturally acquired immunity on virulence gene expression. PLoS Pathog 15:e1007906. https://doi.org/10.1371/journal.ppat.1007906.

77. Petter M, Lee CC, Byrne TJ, Boysen KE, Volz J, Ralph SA, Cowman AF, Brown GV, Duffy MF. 2011. Expression of $P$. falciparum var genes involves exchange of the histone variant H2A.Z at the promoter. PLoS Pathog 7: e1001292. https://doi.org/10.1371/journal.ppat.1001292.

78. Salanti A, Staalsoe T, Lavstsen T, Jensen ATR, Sowa MPK, Arnot DE, Hviid L, Theander TG. 2003. Selective upregulation of a single distinctly structured var gene in chondroitin sulphate A-adhering Plasmodium falciparum involved in pregnancy-associated malaria. Mol Microbiol 49: 179-191. https://doi.org/10.1046/j.1365-2958.2003.03570.x.

79. Liu W, Xie Y, Ma J, Luo X, Nie P, Zuo Z, Lahrmann U, Zhao Q, Zheng Y, Zhao Y, Xue Y, Ren J. 2015. IBS: an illustrator for the presentation and visualization of biological sequences. Bioinformatics 31:3359-3361. https:// doi.org/10.1093/bioinformatics/btv362.
80. Aurrecoechea C, Brestelli J, Brunk BP, Dommer J, Fischer S, Gajria B, Gao X, Gingle A, Grant G, Harb OS, Heiges M, Innamorato F, lodice J, Kissinger JC, Kraemer E, Li W, Miller JA, Nayak V, Pennington C, Pinney DF, Roos DS, Ross C, Stoeckert CJ, Treatman C, Wang H. 2009. PlasmoDB: a functional genomic database for malaria parasites. Nucleic Acids Res 37:D539-D543. https://doi.org/10.1093/nar/gkn814.

81. Sonnhammer EL, von Heijne G, Krogh A. 1998. A hidden Markov model for predicting transmembrane helices in protein sequences. Proc Int Conf Intell Syst Mol Biol 6:175-182.

82. Lord SJ, Velle KB, Mullins RD, Fritz-Laylin LK. 2020. SuperPlots: communicating reproducibility and variability in cell biology. J Cell Biol 219: e202001064. https://doi.org/10.1083/jcb.202001064.

83. Tibúrcio M, Hitz E, Niederwieser I, Kelly G, Davies H, Doerig C, Billker O, Voss TS, Treeck M. 2021. A 39-amino-acid C-terminal truncation of GDV1 disrupts sexual commitment in Plasmodium falciparum. mSphere 6: e01093-20. https://doi.org/10.1128/mSphere.01093-20.

84. Eksi S, Morahan BJ, Haile Y, Furuya $\mathrm{T}$, Jiang $\mathrm{H}$, Ali O, $\mathrm{Xu} \mathrm{H}$, Kiattibutr K, Suri A, Czesny B, Adeyemo A, Myers TG, Sattabongkot J, Su X, Williamson KC. 2012. Plasmodium falciparum gametocyte development 1 (Pfgdv1) and gametocytogenesis early gene identification and commitment to sexual development. Plops Pathog 8:e1002964. https://doi.org/10.1371/journal .ppat.1002964. 